\title{
Genome Sequence and Architecture of the Tobacco Downy Mildew Pathogen Peronospora tabacina
}

\author{
Lida Derevnina, ${ }^{1}$ Sebastian Chin-Wo-Reyes, ${ }^{1}$ Frank Martin, ${ }^{2}$ Kelsey Wood, ${ }^{1}$ Lutz Froenicke, ${ }^{1}$ \\ Otmar Spring, ${ }^{3}$ and Richard Michelmore ${ }^{1}$ \\ ${ }^{1}$ Genome Center, University of California Davis, Davis, CA, U.S.A.; ${ }^{2}$ United States Department of Agriculture-Agricultural \\ Research Service, Salinas, CA U.S.A.; ${ }^{3}$ Institute of Botany, University of Hohenheim, Germany
}

Submitted 22 May 2015. Accepted 12 July 2015.

\begin{abstract}
Peronospora tabacina is an obligate biotrophic oomycete that causes blue mold or downy mildew on tobacco (Nicotiana tabacum). It is an economically important disease occurring frequently in tobacco-growing regions worldwide. We sequenced and characterized the genomes of two $P$. tabacina isolates and mined them for pathogenicity-related proteins and effectorencoding genes. De novo assembly of the genomes using Illumina reads resulted in 4,016 $\left(63.1 \mathrm{Mb}, \mathrm{N}_{50}=79 \mathrm{~kb}\right)$ and 3,245 $\left(55.3 \mathrm{Mb}, \mathrm{N}_{50}=61 \mathrm{~kb}\right)$ scaffolds for isolates 968-J2 and 968-S26, respectively, with an estimated genome size of $68 \mathrm{Mb}$. The mitochondrial genome has a similar size (approximately $43 \mathrm{~kb}$ ) and structure to those of other oomycetes, plus several minor unique features. Repetitive elements, primarily retrotransposons, make up approximately $24 \%$ of the nuclear genome. Approximately 18,000 protein-coding gene models were predicted. Mining the secretome revealed approximately 120 candidate RxLR, six CRN (candidate effectors that elicit crinkling and necrosis), and 61 WY domain-containing proteins. Candidate RxLR effectors were shown to be predominantly undergoing diversifying selection, with approximately $57 \%$ located in variable gene-sparse regions of the genome. Aligning the $P$. tabacina genome to Hyaloperonospora arabidopsidis and Phytophthora spp. revealed a high level of synteny. Blocks of synteny show gene inversions and instances of expansion in intergenic regions. Extensive rearrangements of the gene-rich genomic regions do not appear to have occurred during the evolution of these highly variable pathogens. These assemblies provide the basis for studies of virulence in this and other downy mildew pathogens.
\end{abstract}

Oomycetes are a group of diverse organisms of the Straminipila comprising pathogens that infect both plants and animals. While they morphologically resemble fungi, oomycetes are taxonomically more closely related to brown algae, diatoms, and Labyrinthulomycota with which they form the Heterokontobionta (Bartnicki-Garcia 1968; Thines 2014; Spring 2012). Worldwide, plant-pathogenic oomycetes cause some

Genomic sequences have been deposited in the GenBank database under the accession number PRJNA285243. The mitochondrial genome was deposited in GenBank (GenBank accessions KT893455 and KT893456).

Corresponding author: R. Michelmore;

E-mail: rwmichelmore@ucdavis.edu; Telephone: +1.530.752.8889; Fax: +1.530.754.9658.

*The $\boldsymbol{e}$-Xtra logo stands for "electronic extra" and indicates that seven supplementary figures and 15 supplementary tables are published online.

@ 2015 The American Phytopathological Society of the most devastating diseases of crops, ornamentals, and native species, leading to yield loss and damage to agricultural and natural ecosystems (Jiang and Tyler 2012). Some of the most destructive oomycete pathogens occur within the order Peronosporales. Members of this order have diverse lifestyles ranging from necrotrophy (Pythium spp.) to hemibiotrophy (Phytophthora spp.) and obligate biotrophy (downy mildews) (Thines 2014). Downy mildews constitute the largest taxonomic group within the Peronosporales, with more than 700 known species and include members from the economically important genera Bremia (Michelmore and Wong 2008), Plasmopara (Burruano 2000), Peronospora (Voglmayr 2003), Peronosclerospora (Perumal et al. 2008), and Sclerospora (Sastry et al. 1997) that infect a broad range of wild and crop plants (Spencer 1981). Individual species of downy mildew generally have narrow host ranges, making them ideal for studying the evolutionary and molecular basis for host specialization in plant-pathogen interactions.

The advent of next-generation sequencing methods, with their reduced costs and high throughput, offer unprecedented opportunities to sequence and perform comparative analyses of a large number of genomes. Several genome sequences for plant-pathogenic oomycetes are now available, and much of the current understanding of the mechanisms and evolution of virulence in oomycetes have been based on genome and transcriptome sequencing efforts. Twelve phytopathogenic species belonging to the peronosporalean lineage have so far been sequenced and annotated; these comprise four species of hemibiotrophic Phytophthora spp. (Phytophthora infestans, Phytophthora sojae, Phytophthora ramorum, and Phytophthora capsici) (Haas et al. 2009; Lamour et al. 2012; Tyler et al. 2006), seven Pythium spp. (Adhikari et al. 2013; Lévesque et al. 2010), and one obligate biotroph Hyaloperonospora arabidopsidis (Baxter et al. 2010). A draft genome assembly of Pseudoperonospora cubensis (Tian et al. 2011) and the transcriptome of Bremia lactucae (Stassen et al. 2012) are also available. Given the paucity of downy mildew sequences, there is a great need and opportunity for sequencing of the genomes of additional downy mildews to provide insights into the basis of virulence of these important biotrophic pathogens.

Peronospora tabacina is an obligate biotrophic oomycete that causes downy mildew, also known as blue mold of tobacco (Nicotiana tabacum) (McKeen 1989). It is an economically important pathogen that occurs frequently in tobacco-growing regions worldwide; although epidemics are irregular and highly dependent on conducive cool wet weather conditions (Spring et al. 2013). The disease occurs annually in Europe and has become widespread in tobacco-growing regions of North America (Sukno et al. 2002; Ristaino et al. 2007). Control of the pathogen is 
challenging because few chemical measures are available and isolates of $P$. tabacina that are insensitive to metalaxyl are becoming increasingly widespread (Bruck et al. 1982; Wiglesworth et al. 1988; Zipper et al. 2009). Although sexual reproduction through oospores can occur (Heist et al. 2002), the establishment and development of populations in the United States and Europe are predominantly due to asexual/clonal propagation (Sukno et al. 2002; Spurr and Todd 1919; Zipper et al. 2009). Except for a study on the diversity of microsatellite loci (Trigiano et al. 2012), little has been reported to date on the genome structure, genetic diversity, and mechanisms of virulence of P. tabacina, in part, due to the previous difficulties of characterizing a biotrophic pathogen.

Biotrophic pathogens secrete a variety of proteins that facilitate infection of their hosts. Oomycetes secrete a set of pathogenicity-related proteins into the plant apoplast, in which they interact with extracellular targets and cellsurface receptors of the host; these include small cysteine-rich proteins, elicitins, proteases and necrosis and ethylene inducing proteins (NEPs) (Kamoun 2006). Other secreted pathogenicity-related proteins are translocated into the plant cell; these cytoplasmic effectors fall into two classes that have been extensively characterized in oomycetes: those with a RxLR motif and those with a LxLFLAK motif (crinkling and necrosis-inducing [CRN]) (Kamoun 2006). Effectors are key determinants of virulence and have been shown to modulate the host defense system and enable tissue colonization (Rafiqi et al. 2012). In some cases, host resistance genes ( $R$ genes) recognize effector proteins and elicit a resistance response that may involve host cell death (hypersensitive response). Such effectors are deemed to have avirulence activity and are often designated as encoded by avirulence genes. Characterization of additional pathogen effectors, their targets, and their cognate $R$ genes will expand the understanding of host-pathogen interactions and facilitate implementation of effective control strategies.

In order to investigate the genome structure, genetic variability, and the repertoire of candidate pathogenicity-related proteins in $P$. tabacina, two single-sporangiophore strains differing in phenotype, aggressiveness, and fungicide resistance (Spring et al. 2013; Zipper et al. 2009) were sequenced and their nuclear and mitochondrial genomes were assembled. This revealed a small nuclear genome with low complexity relative to other members of the Peronosporales. Comparative structural genomics on $P$. tabacina and other oomycetes uncovered extensive synteny between species. Large repertoires of genes encoding pathogenicity-related proteins were identified in both isolates. The overall structure of the mitochondrial genome was similar to those of other oomycetes along with several minor unique features. This high-quality draft genome sequence provides the basis for further studies on variability and virulence on P. tabacina.

\section{RESULTS}

\section{Genome characterization.}

The sequences of two P. tabacina strains (968-J2 and 968S26) were generated on an Illumina MiSeq platform. Two strains were sequenced to provide cross-validation of the assemblies, identify species-specific characteristics, and enable intraspecific comparisons. After trimming and filtering of the sequence data to remove small or bad-quality as well as bacterial sequences, 35.5 and $17.1 \mathrm{~Gb}$ of high-quality reads were obtained for the two isolates, providing $128.9 \times$ and $61.8 \times$ coverage of $968-\mathrm{J} 2$ and $968-\mathrm{S} 26$, respectively, with an estimated genome size of $68 \mathrm{Mb}$. The genomes were assembled using both the ALLPaths-LG and CLC programs. Assembly with ALLPaths-LG resulted in fewer contigs with larger $\mathrm{N}_{50}$ values (Yandell and Ence 2012) than with CLC; however, the CEGMA score (Parra et al. 2007) indicated that the former assembly was less comprehensive (Table 1, Supplementary Table S1). In order to capture the most complete representation of the genome, the ALLPaths-LG assembly was used as the base for the genome sequence, due to its longer and fewer scaffolds; this was supplemented with contigs from the CLC assembly that were not present in the ALLPaths-LG assembly, and the hybrid assembly was scaffolded with SSPACE (Boetzer et al. 2011). This resulted in high-quality assemblies for both isolates, with 4,016 and 3,245 scaffolds representing 63.1 and $55.3 \mathrm{Mb}$ of assembled sequence for isolates $968-\mathrm{J} 2$ and 968S26, respectively (Table 1 ).

The level of heterozygosity within each genome was calculated by counting the number of single nucleotide polymorphisms (SNP) that exist between the two $P$. tabacina genomes using SAMTOOLs (Li et al. 2009). SNP analysis revealed that at least $32 \%$ of each genome was heterozygous (Supplementary Fig. S1). Read depth analyses showed that approximately $82 \%$ of the two haplotypes were merged in the assembly, with approximately $10 \mathrm{Mb}$ of the assembly representing either one or the other haplotype.

The timing of outcrossing was estimated twice by independently calculating the levels of heterozygosity in all predicted genes as well as in approximately 405 eukaryotic orthologous group (KOG) sequences based on the frequency of heterozygous SNP. This revealed heterozygosity levels of approximately $25 \%$ for both gene sets in each strain. Therefore, assuming that each generation of selfing reduces heterozygosity by half, both strains have most likely undergone an outcrossing event, followed by no more than two generations of selfing (Supplementary Table S2).

\section{Nuclear repeat sequences.}

Repetitive DNA (including low-complexity sequences) were identified de novo using RepeatModeler and represent approximately $24 \%$ of the genome (Table 2 ; Supplementary Table $\mathrm{S} 3)$. This proportion of repeated sequences is comparable to

Table 1. The ALLPaths-LG and CLC assemblies and annotation statistics for two genomes of Peronospora tabacina

\begin{tabular}{|c|c|c|c|c|c|c|}
\hline & \multicolumn{3}{|c|}{ 968-J2 assemblies } & \multicolumn{3}{|c|}{ 968-S26 assemblies } \\
\hline & ALLPaths-LG & CLC $^{\mathrm{a}}$ & Hybrid & ALLPaths-LG & CLC $^{\mathbf{a}}$ & Hybrid \\
\hline Contig total length $(\mathrm{Mb})$ & 30.3 & 41.2 & 45.9 & 33.5 & 37.1 & 44.8 \\
\hline Number of contigs & 6,763 & 16,053 & 10,648 & 6,399 & 13,977 & 8,429 \\
\hline N50 contig length & 11,219 & 4,201 & 11,413 & 13,645 & 1,351 & 15,177 \\
\hline Scaffold total (Mb) & 43.9 & - & 63.1 & 42.2 & - & 55.3 \\
\hline Number of scaffolds & 1,796 & - & 4,016 & 2,012 & - & 3,245 \\
\hline N50 scaffold length & 83,266 & - & 78,959 & 61,125 & - & 60,814 \\
\hline GC content $(\%)$ & 48 & 48 & 48 & 48 & 47 & 48 \\
\hline CEGMA ( $\%$ full, $\%$ partial) & $77.02,86.29$ & $87.9,93.95$ & $92.34,95.16$ & $82.66,87.9$ & $87.9,94.76$ & $92.34,95.16$ \\
\hline
\end{tabular}

\footnotetext{
a Scaffolds were not generated by CLC.
} 
Phytophthora ramorum (28\%) and Phytophthora capsici (19\%), which have similar small genomes of 65 and $64 \mathrm{Mb}$, respectively, relative to other Phytophthora spp. (Lamour et al. 2012; Tyler et al. 2006). The repeated elements were classified into known classes of transposons, revealing that the majority of repetitive sequences were retrotransposons (about 58\%) with only a small portion of DNA transposons (about 5\%), the remaining about $37 \%$ were not classified. The most abundant repeats are long-terminal repeat elements, of which about $38 \%$ are Gypsy-like elements and 19\% are Copia-like elements (Fig. 1).

Long-terminal repeat retrotransposon (LTR-RT) analysis. The ages of retrotransposon insertion events was calculated based on the sequence divergence of their LTRs. The number of clusters and the number of classifications were similar for each

Table 2. Proportion of repeats in the genomes of two isolates of Peronospora tabacina $^{\text {a }}$

\begin{tabular}{lcc}
\hline Repeat element & 968-J2 & 968-S26 \\
\hline Total & $37,595 / 22.2 \%$ & $36,730 / 25.71 \%$ \\
DNA transposons & $1,444 / 1 \%$ & $1,435 / 1 \%$ \\
Retrotransposons & $15,105 / 13 \%$ & $14,738 / 15 \%$ \\
Unknown & $21,046 / 8 \%$ & $20,557 / 9 \%$ \\
\hline
\end{tabular}

${ }^{a}$ Results for hybrid assemblies. Presented as repeat elements/\% of the genome masked. isolate; however, more recent retrotransposon activity was indicated in the Gypsy family of strain 968-S26 as compared with 968-J2 (Fig. 1).

\section{Annotation.}

The potential gene content was investigated in three ways. Gene models were generated using multiple runs of MAKER. Annotation of the two isolates using MAKER led to the prediction of 20,968 (968-J2) and 20,460 (968-S26) proteincoding gene models (Supplementary Table S4; Supplementary Figs. S2 and S3). These were filtered based on alignment of known gene model structures and transcript evidence (968-J2: 18,447; 968-S26: 17,678). They were then collapsed into 11,310 (968-J2) and 10,707 (968-S26) loci by removing overlapping gene models. The gene statistics were similar for the two isolates. The average gene length was approximately $1,250 \mathrm{bp}$, with a mean of 2.3 introns per gene and average intron lengths of approximately $113 \mathrm{bp}$. Even though the mean intron number per gene was low, a few intron-rich genes were predicted with as many as 20 introns. The average predicted exon length was approximately $510 \mathrm{bp}$ with a maximum length of approximately $15 \mathrm{~kb}$. InterPro identified protein domains in about $15,000(82 \%)$ of the predicted genes. Protein clustering using OrthoMCL grouped 17,171 genes into 3,980 families



Fig. 1. Age distribution of long-terminal repeat retrotransposon (LTR-RT) insertions in strains 968-J2 and $968-$ S26 of Peronospora tabacina. The top panels show the distribution of insertion estimates of LTR clustered by family, where the corresponding value represents the number of members in each family. The bottom panels show the same, with the $x$ axis representing the estimated time of insertion of the LTR-RT superfamilies. RLG = retrotransposon LTR Gypsy; RLC = retrotransposon LTR Copia. 
with 1,276 singletons in isolate 968-J2 and 16,334 genes into 3,801 families with 1,344 singletons in isolate $968-S 26$. None of the 10 largest annotated protein families are obviously involved in pathogenesis-related processes (Supplementary Table S5).

Over- and underrepresented gene models. Predicted gene models were clustered into gene families, based on protein similarity across the oomycetes (two P. tabacina strains, Hyaloperonospora arabidopsidis, Phytophthora infestans, Phytophthora ramorum, and Phytophthora sojae), using OrthoMCL. A total of 17,712 gene families were identified, of which 1,649 were unique to the $P$. tabacina clade. Grouping gene families by phylogeny (Fig. 2) revealed that 7,742 gene families were shared between the downy mildew and the Phytophthora species. A total of 5,693 core genes were observed within the downy mildews. Six gene-family clusters were shown to be significantly different between $P$. tabacina and Phytophthora spp. (Supplementary Table S6); four genefamily clusters were overrepresented (reverse transcription domain, myosin domain, Ras proteins, and heat-shock chaperonin-binding motif) in P. tabacina compared the Phytophthora spp. and two were underrepresented (ABC transporters and the ATPase family associated with various cellular activities) in P. tabacina compared with Phytophthora spp.

Predicted gene models were also clustered based on functional annotation information and were grouped according to domains. A total of 138 protein domains differed significantly in abundance between the $P$. tabacina strains and the three Phytophthora spp. (Supplementary Table S7); 48 protein domains were underrepresented in $P$. tabacina compared with Phytophthora spp. and 90 were overrepresented. Several proteins potentially involved in pathogenicity differed between the two genera; such proteins tended to be underrepresented in $P$. tabacina, including elicitin-like and NPP1-like proteins (Table 3).

\section{Repertoires of pathogenicity-related secreted proteins.}

Like many other oomycete pathogens, $P$. tabacina likely secretes effector proteins as well as degradative enzymes that alter host physiology and facilitate colonization. Since effector proteins have generally been identified in repeat-rich regions of the genome (Haas et al. 2009) and MAKER-predicted gene models often select against these regions (Cantarel et al. 2008), a six-frame translation was used to identify effector proteins. The six-frame translation was shown to be more inclusive than the predicted gene models (Fig. 3), and totals of 974,757 and 947,578 potential open reading frames (ORF) were independently determined for $968-\mathrm{J} 2$ and $968-\mathrm{S} 26$. Of the total potential ORF, approximately 61,000 ORF encode at least 80 aa (less than $10 \%$ X's) and approximately 3,000 (5\%) were predicted to be secreted using SignalP. Genes encoding predicted secreted proteins from both strains were clustered using OrthoMCL, revealing 2,173 clusters containing 4,976 secreted proteins and 1,052 singletons. The largest gene family contained six members and encoded $\beta$-glucan synthesis-associated proteins (SKN1), involved in cell-wall synthesis. Other families of secreted proteins include protease inhibitors (aspartyl, serine, and cysteine), elicitin and elicitin-like proteins, NPP1-like proteins, E3 ubiquitin ligases, and cell wall-degrading enzymes (pectinesterase, pectin lyase, phospholipase) (Table 4).

The largest and most diverse effector protein family in the Peronosporales possesses a modular domain structure consisting

Table 3. Protein domains in predicted gene models potentially involved in pathogenicity that were under- and overrepresented in two Peronospora tabacina strains as compared with three Phytophthora spp. (Phytophthora infestans, Phytophthora ramorum, Phytophthora sojae)

\begin{tabular}{lc}
\hline Protein domains $^{\mathbf{a}}$ & $\boldsymbol{P}$ value \\
\hline Underrepresented in $P$. tabacina & \\
ABC transporters (84/164) & $9.8 \mathrm{E}-10$ \\
Aspartyl protease families (14/250) & $3.0 \mathrm{E}-72$ \\
Kazal-type domain (serine-protease inhibitor) & $1.7 \mathrm{E}-06$ \\
$\quad(6 / 27)$ & $5.2 \mathrm{E}-11$ \\
Elicitin-like proteins (18/62) & $6.9 \mathrm{E}-15$ \\
Pectate lyase (2/38) & $4.0 \mathrm{E}-06$ \\
NPP1-like (necrosis-inducing proteins) (17/44) & $1.0 \mathrm{E}-09$ \\
Phospholipase D (36/89) & $1.0 \mathrm{E}-06$ \\
Phosphatidylinositol-specific phospholipase & \\
$\quad(2 / 19)$ & $1.8 \mathrm{E}-08$ \\
Overrepresented in $P$. tabacina & $9.6 \mathrm{E}-06$ \\
$\quad$ Serine/threonine phosphatase family (450/336) \\
Peptidase family C50 (12/3)
\end{tabular}
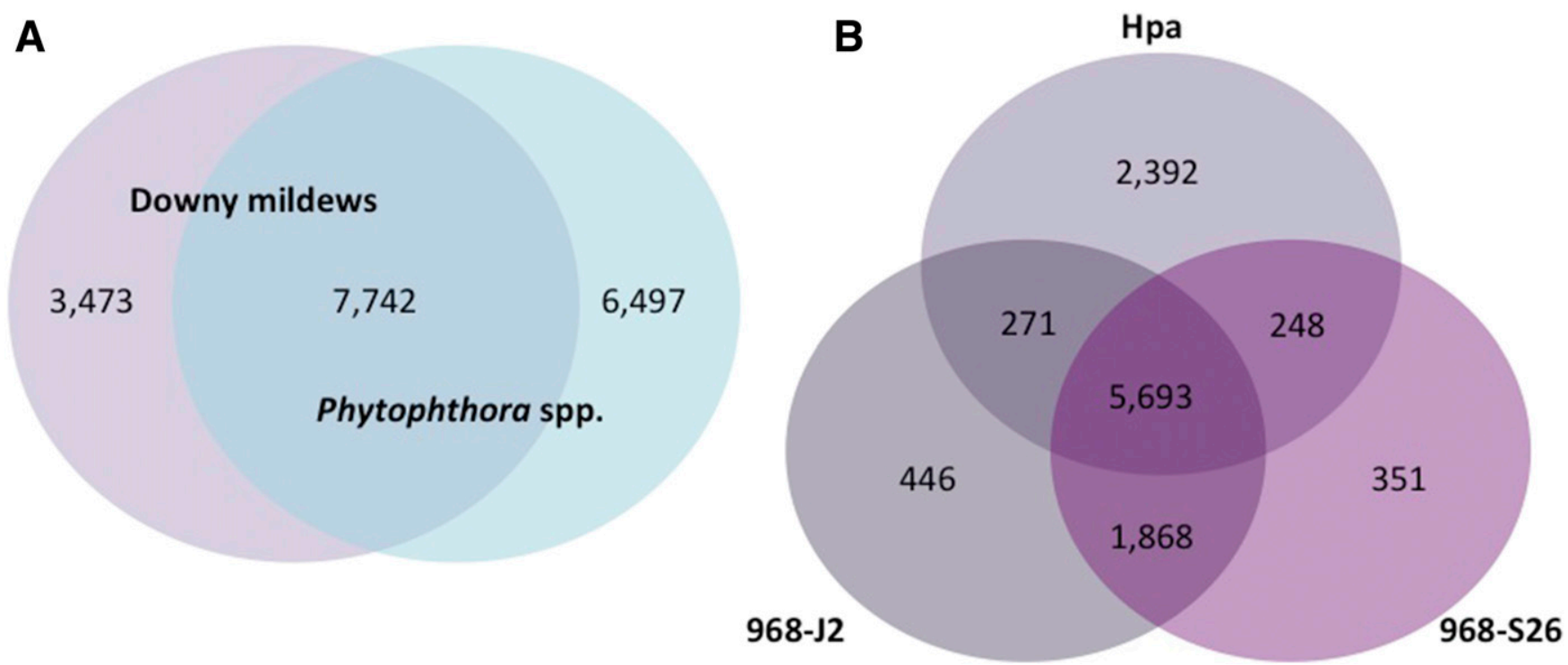

Fig. 2. OrthoMCL gene families shared among different oomycete species. A, Gene families shared between downy mildews (strains $968-\mathrm{J} 2$ and $968-\mathrm{S} 26$ of Peronospora tabacina and Hyaloperonospora arabidopsidis combined) and Phytophthora spp. (Phytophthora infestans, Phytophthora sojae, and Phytophthora ramorum combined). B, Gene families shared between tobacco and Arabidopsis downy mildews. Hpa $=H$. arabidopsidis. 
of an amino-terminal (RxLR-EER) motif involved in translocation and a carboxyl-terminal effector domain (Lamour et al. 2007). Phytophthora genomes encode hundreds of potential effector proteins (350 to 563) with RxLR cell-entry motifs that have been implicated in pathogenesis (Haas et al. 2009; Jiang et al. 2008; Kale et al. 2010; Whisson et al. 2007). Two different bioinformatic approaches were used to mine the $P$. tabacina secretome for candidate RxLR effectors. Hidden Markov model (HMM)-based searches identified approximately 44 RxLR candidates; additionally, about 120 candidates were identified in each strain using string searches (Table 5). Similar numbers of candidate RxLR effector-encoding genes, 122 in 968-J2 and 117 in 968-S26, remained after filtering based on length ( $\geq 50$ aa $C$ terminal to the RxLR motif) and the removal of redundant sequences. Forty-six of these were identical at the amino acid level among the two strains. These sequences
Table 5. Number of candidate pathogenicity-related proteins identified in Peronospora tabacina

\begin{tabular}{lcc}
\hline Function & 968-J2 & 968-S26 \\
\hline All RxLR motif containing proteins ${ }^{\mathrm{a}}$ & $171(44)$ & $161(45)$ \\
Filtered $<50$ aa C terminal to RxLR & $43(2)$ & $42(0)$ \\
Filtered $\geq 50$ aa C terminal to RxLR ${ }^{\mathrm{a}}$ & $128(42)$ & $122(45)$ \\
Filtered $\geq 50$ aa with no redundants & \\
All WY domain-containing proteins with & $122(40)$ & $117(44)$ \\
open reading frames (ORF) $\geq 80$ aa & 150 & 160 \\
Predicted in the secretome & 63 & 60 \\
RxLR ( $\geq 50$ aa C terminal) & 28 & 28 \\
CRN (crinkling and necrosis-inducing) & 120 & 147 \\
proteins, ORF $\geq 80$ aa & & \\
Predicted in the secretome & 4 & 10 \\
\hline${ }^{a}$ Candidate RxLR effectors, identified using hidden & Markov model \\
searches, are in parentheses. &
\end{tabular}

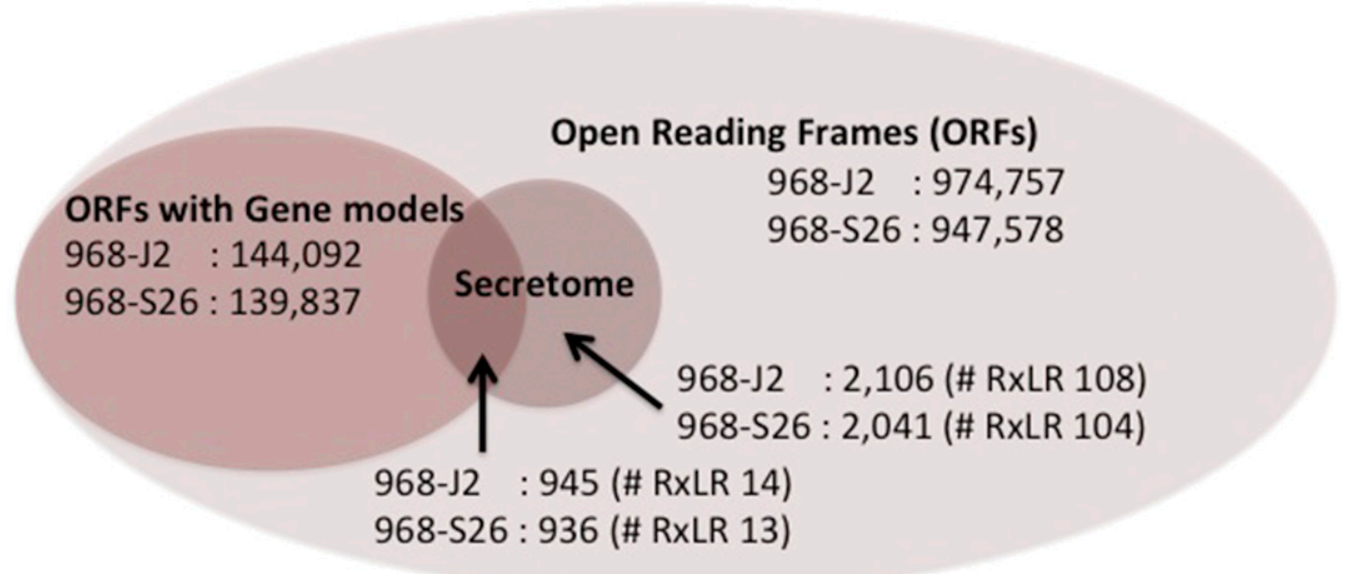

Fig. 3. The Peronospora tabacina entire open reading frame set, unfiltered, showing the overlap between the predicted gene models and the secretome datasets

Table 4. Numbers of gene models in protein families implicated in pathogenesis in the genome assemblies of three downy mildews and three Phytophthora species

\begin{tabular}{|c|c|c|c|c|c|c|}
\hline & \multicolumn{2}{|c|}{ Peronospora tabacina ${ }^{a}$} & \multirow[b]{2}{*}{$\mathrm{Hpa}^{\mathrm{b}}$} & \multicolumn{3}{|c|}{ Phytophthora spp. ${ }^{\mathrm{c}}$} \\
\hline & 968-J2 & 968-S26 & & Phi & Phs & Phr \\
\hline $\mathrm{ABC}$ transporters & $85(2)$ & $76(4)$ & 65 & 170 & 188 & 192 \\
\hline Cytochrome P450 & $18(1)$ & $14(1)$ & 15 & 30 & 39 & 31 \\
\hline Cutinase (IPR000675) & 0 & 0 & 2 & 4 & 15 & 4 \\
\hline Aspartyl protease families (GO:0004190) & $42(4)$ & $26(4)$ & 60 & 23 & 75 & 47 \\
\hline $\begin{array}{l}\text { Cysteine protease families (IPR000668| } \\
\text { IPR001096|IPR001300|IPR003323) }\end{array}$ & $43(11)$ & $42(7)$ & 23 & 37 & 36 & 39 \\
\hline Serine protease & $42(11)$ & $47(10)$ & 30 & 96 & 111 & 75 \\
\hline Elicitin-like proteins (IPR002200) & $15(12)$ & $13(9)$ & 17 & 43 & 70 & 57 \\
\hline Glycosyl hydrolase & $96(22)$ & $88(20)$ & 72 & 179 & 202 & 185 \\
\hline Lipases & $25(2)$ & $36(5)$ & 17 & 27 & 44 & 26 \\
\hline Peptidase activity (GO:0008233) & $36(1)$ & $23(1)$ & 13 & 11 & 16 & 17 \\
\hline Transmembrane transport (GO:0055085) & $301(5)$ & $252(7)$ & 156 & 317 & 397 & 316 \\
\hline Cellulose binding (GO:0030248) & 0 & 0 & 0 & 8 & 10 & 13 \\
\hline NPP1-like (IPR008701) & $24(13)$ & $15(13)$ & 21 & 45 & 81 & 69 \\
\hline Pectinesterase (GO:0030599) & $4(3)$ & $3(2)$ & 3 & 11 & 20 & 13 \\
\hline Pectin lyase (IPR012334) & $8(4)$ & 7 (3) & 23 & 92 & 113 & 74 \\
\hline Phospholipase D & $29(2)$ & $24(2)$ & 11 & 24 & 30 & 23 \\
\hline Ubiquitin E3 ligase activity (GO:0008270) & $759(15)$ & 735 (12) & 663 & 625 & 918 & 769 \\
\hline Cysteine-rich secretory protein (IPR014044) & $26(14)$ & $27(15)$ & 13 & 29 & 42 & 28 \\
\hline CBEL (Phytophthora infestans ACM68430) ${ }^{\mathrm{d}}$ & $2(4)$ & $7(6)$ & 4 & 31 & 38 & 30 \\
\hline
\end{tabular}

${ }^{a}$ The numbers in parentheses refers to protein families observed in the secretomes of Peronospora tabacina. The P. tabacina secretome was identified as possessing an amino terminal signal peptide in all possible open reading frames that encoded 80 or more amino acids from both strands of the genomic DNA sequence.

${ }^{\mathrm{b}} \mathrm{Hpa}=$ Hyaloperonospora arabidopsidis (v7.01, id3073). Gene models were predicted using the MAKER v2.10 annotation pipeline. Predicted gene models were functionally annotated using InterProscan v5 and clustered into gene families using OrthoMCL v2.0.7.

${ }^{\mathrm{c}} \mathrm{Phi}=$ Phytophthora infestans $(\mathrm{v} 1, \mathrm{id} 3072) ; \mathrm{Phs}=$ Phytophthora sojae (v2, id16187); and Phr = Phytophthora ramorum $(\mathrm{v} 1.1, \mathrm{id} 3074)$.

${ }^{\mathrm{d}}$ CBEL (cellulose binding elicitor lectin) annotation terms: IPR00177, IPR000254, IPR003014, IPR029469, IPR003609, IF0024, PF14295, SM00223, SM00236, PS50948. 
were functionally annotated using the CD-search program, which searches for conserved domains. The largest family was comprised of 39 (968-J2) and 49 (968-S26) genes predicted to encode members of the serine endopeptidase-S8 family (Rawlings et al. 2010). Using OrthoMCL, 70 out of the 122 and 78 out of the 117 RxLR candidates were grouped into 11 and 12 clusters in strains 968-J2 and 968-S26, respectively. Clustering predicted RxLR proteins of $P$. tabacina with those of $H$. arabidopsidis, Phytophthora infestans, Phytophthora ramorum, and Phytophthora sojae revealed that only one cluster was common between all five species (Supplementary Fig. S5; Supplementary Table S8). Many of the RxLR proteins from the three Phytophthora spp. clustered together, while most of the $P$. tabacina RxLR proteins were private to the species.

HMM searches of the predicted proteomes yielded 150 (968-

J2) and 160 (968-S26) putative WY domain-containing proteins

A



Fig. 4. A, Cladogram of predicted WY domain-containing proteins in the secretome of Peronospora tabacina. For each protein, branches are color-coded by strain of origin (968-J2 in purple, 968-S26 in green), circles indicate presence (black) or absence (white) of an N-terminal RXLR motif, and bar lengths indicate the number of WY domains predicted for each protein. B, Histograms showing the distribution of proteins by number of WY domains per protein for the two strains of P. tabacina. 
(Table 5; Supplementary Table S9). Of these, 63 (968-J2) and 60 (968-S26) were predicted by SignalP to be secreted and approximately half possessed an N-terminal RxLR motif (Table 5). The candidate WY effectors contained a variable number of WY domains per protein, with most proteins possessing one to three WY domains, a few with five to eight domains, and one protein with 18 predicted WY domains (Fig. $4 \mathrm{~A}$ and $\mathrm{B}$ ). Neighbor-joining analysis using multiple sequence alignment of WY amino-acid sequences revealed that, while most candidate WY effectors are present in both strains of $P$. tabacina, there are several groups that appear to show expansion in one strain or the other (Fig. 4A). While most of the closest neighbors in the similarity tree have the same number of WY domains, there are several transitions that suggest gain of a single WY domain or duplication of the number of WY domains during effector evolution (Fig. 4A).

In addition to RxLR effectors and WY domain-containing proteins, the genomes of many oomycete pathogens encode a large repertoire of candidate $\mathrm{CRN}$ effectors that elicit crinkling and necrosis in planta, termed 'crinklers' (Haas et al. 2009; Torto et al. 2003; Win and Kamoun 2008). CRN were first identified in Phytophthora species based on their ability to elicit plant cell death and have, so far, been identified in all phytopathogenic oomycete genomes sequenced to date (Adhikari et al. 2013; Baxter et al. 2010; Haas et al. 2009; Kemen et al. 2011; Lévesque et al. 2010; Tyler et al. 2006). A total of 120 (968-J2) and 147 (968-S26) putative CRN proteins were predicted from the 61,000 predicted ORF (minimum of $80 \mathrm{aa}$ ) sequences. Of these, only four $(3 \%, 968-\mathrm{J} 2)$ and $10(7 \%, 968-$ S26) were predicted to possess a signal peptide using SignalP (Table 5).

Detection of positive selection acting on candidate RxLR and WY domain-containing effector proteins. Most proteins are under purifying selection that results in conservation of the amino-acid sequence and preservation of function. The genome-wide average $\mathrm{d}_{\mathrm{N}}: \mathrm{d}_{\mathrm{S}}$ (nonsynonymous to synonymous) ratio for 874 syntenic genes between the two strains of $P$. tabacina was calculated using CoGe to be 0.03 . To investigate whether positive selection was acting on candidate RxLR and WY domain-containing effectors, 104 allele pairs were identified through bidirectional BLASTP searches of the two $P$. tabacina strains, of which 26 had differences in their nucleotide sequences that allowed calculation of $\mathrm{d}_{\mathrm{N}}: \mathrm{d}_{\mathrm{S}}$ ratios $(\omega)$ (Table 6). Calculation of $\omega$ across the entire length of the candidate effector proteins revealed that $\mathrm{d}_{\mathrm{N}}$ was significantly greater than $d_{S}$ (average $\omega=1.89$ ) in 14 of these 26 pairs. To test whether selection had acted differently on the different domains of the candidate effector proteins, the sequences were partitioned into $\mathrm{N}$-terminal and $\mathrm{C}$-terminal regions. The proteins were split into two equal fragments based on the amino acid alignment of the allelic pairs. More pairs exceeded the entire protein average of $\omega=1.89$ at the C-terminus ( 5 of 26 pairs) than the $\mathrm{N}$-terminus ( 2 of 26 pairs). The average $\omega$ for the N-terminus was 1.11 , with the highest observed $\omega=3.07$ (RXLR38), while the average $\omega$ at the C-terminus was 1.75 , with the highest observed $\omega=5.91$ (WY11) (Table 6). This is consistent with positive selection acting on the C-terminal regions of this subset of candidate effector proteins in P. tabacina; this is similar to observations in Phytophthora spp. (Win et al. 2007).

\section{(In)sensitivity to metalaxyl.}

Plant diseases caused by oomycetes have been controlled using metalaxyl and its enantiopure $\mathrm{R}$ form mefenoxam since the 1980s (Cohen and Coffey 1986). However, insensitive strains have often quickly arisen in multiple oomycete pathogens, rendering the chemical ineffective. The precise biochemical mode of action of metalaxyl remains unclear. One mechanism of action seems to involve the inhibition of ribosomal RNA synthesis by interfering with the activity of the RNA polymerase I complex (Davidse et al. 1983). The molecular basis for insensitivity of some, but not all, metalaxyl-insensitive strains

Table 6. Summary of positive selection analysis on orthologous candidate RxLR and WY domain-containing sequences from two Peronospora tabacina strains, using pair-wise comparisons ${ }^{\mathrm{a}}$

\begin{tabular}{|c|c|c|c|c|c|c|c|c|c|}
\hline \multirow[b]{2}{*}{ Domain } & \multicolumn{3}{|c|}{ Fullseq } & \multicolumn{3}{|c|}{ N-terminus } & \multicolumn{3}{|c|}{ C-terminus } \\
\hline & $\mathbf{d}_{\mathbf{N}}$ & $\mathbf{d}_{\mathbf{S}}$ & $\mathbf{d}_{\mathbf{N}} / \mathbf{d}_{\mathrm{S}}$ & $\mathbf{d}_{\mathbf{N}}$ & $\mathbf{d}_{\mathbf{S}}$ & $\mathbf{d}_{\mathrm{N}} / \mathbf{d}_{\mathrm{S}}$ & $\mathbf{d}_{\mathbf{N}}$ & $\mathbf{d}_{\mathrm{S}}$ & $\mathbf{d}_{\mathrm{N}} / \mathbf{d}_{\mathrm{S}}$ \\
\hline WY 2 & 0.0684 & 0.0335 & 2.0421 & 0.1442 & 0.0699 & 2.0627 & 0 & 0 & 0 \\
\hline WY 4 & 0.8102 & 1.0776 & 0.7519 & 0.8072 & 1.1716 & 0.689 & 0.8245 & 0.988 & 0.8345 \\
\hline WY 5 & 0.01 & 0.006 & 1.6712 & 0 & 0 & 0 & 0.0197 & 0.0131 & 1.5016 \\
\hline WY 8 & 0.0028 & 0.0026 & 1.1026 & 0.0056 & 0.0054 & 1.0276 & 0 & 0 & 0 \\
\hline WY 9 & 0.0094 & 0.0115 & 0.8166 & 0 & 0 & 0 & 0.0185 & 0.0242 & 0.7639 \\
\hline WY 11 & 0.0655 & 0.0335 & 1.9557 & 0.0846 & 0.0563 & 1.5025 & 0.0479 & 0.0081 & 5.9077 \\
\hline WY 12 & 0.006 & 0.0067 & 0.8928 & 0.0021 & 0 & 99 & 0.0098 & 0.016 & 0.6122 \\
\hline WY 16 & 0.0039 & 0.0135 & 0.2919 & 0.0042 & 0.0245 & 0.1708 & 0.0032 & 0 & 99 \\
\hline RXLR_WY22 & 0.0011 & 0.0037 & 0.3044 & 0.0022 & 0.0078 & 0.2867 & 0 & 0 & 0 \\
\hline RXLR 23 & 0.0455 & 0.0298 & 1.524 & 0.0529 & 0.0339 & 1.5588 & 0.0386 & 0.0244 & 1.5828 \\
\hline RXLR_WY25 & 0.0078 & 0.0108 & 0.7227 & 0.0155 & 0.0224 & 0.6923 & 0 & 0 & 0 \\
\hline RXLR2̄8 & 0.0101 & 0.0082 & 1.2318 & 0 & 0 & 0 & 0.0204 & 0.0163 & 1.2531 \\
\hline RXLR32 & 0.0007 & 0.0039 & 0.1841 & 0 & 0.0015 & 0 & 0.0016 & 0.0056 & 0.2912 \\
\hline RXLR35 & 0.1 & 0.0273 & 3.6665 & 0.0072 & 0.0001 & 99 & 0.2131 & 0.0618 & 3.4451 \\
\hline RXLR_WY36 & 0.0048 & 0.0029 & 1.64 & 0 & 0 & 0 & 0.0101 & 0.0056 & 1.8014 \\
\hline RXLR3̄38 & 0.1037 & 0.0529 & 1.96 & 0.0342 & 0.0111 & 3.0718 & 2.4929 & 2.9986 & 0.8314 \\
\hline RXLR39 & 0.1434 & 0.0916 & 1.5653 & 0.0124 & 0.0264 & 0.4677 & 0.3453 & 0.1345 & 2.567 \\
\hline RXLR42 & 0.0395 & 0.0119 & 3.3241 & 0.0154 & 0.0002 & 99 & 0.1019 & 0.0348 & 2.9296 \\
\hline RXLR43 & 0.0831 & 0.035 & 2.3749 & 0.1273 & 0.0555 & 2.2938 & 0.0463 & 0.0145 & 3.1825 \\
\hline RXLR44 & 0.0041 & 0.0118 & 0.3503 & 0.008 & 0.0233 & 0.3449 & 0 & 0 & 0 \\
\hline RXLR_WY46 & 0.0187 & 0.0221 & 0.8455 & 0.0066 & 0.0168 & 0.3955 & 0.0308 & 0.0271 & 1.1371 \\
\hline RXLR 47 & 0.0026 & 0.016 & 0.1648 & 0 & 0.0241 & 0.001 & 0.0053 & 0.0064 & 0.825 \\
\hline RXLR_WY49 & 0.0184 & 0.0171 & 1.0755 & 0.0387 & 0.0324 & 1.1934 & 0 & 0 & 0 \\
\hline RXLR5 1 & 0.0025 & 0.0052 & 0.4871 & 0.005 & 0.0001 & 99 & 0 & 0.0102 & 0 \\
\hline RXLR52 & 0.0103 & 0.027 & 0.3812 & 0.0184 & 0.0494 & 0.3717 & 0.0026 & 0 & 99 \\
\hline RXLR_WY53 & 0.0694 & 0.0546 & 1.2715 & 0.1013 & 0.0617 & 1.6411 & 0.0145 & 0.0554 & 0.2609 \\
\hline
\end{tabular}

\footnotetext{
${ }^{a}$ For effector proteins, refer to Supplementary Table S10. $\mathrm{d}_{\mathrm{N}}=$ nonsynonymous; $\mathrm{d}_{\mathrm{S}}=$ synonymous.
} 
of Phytophthora infestans has been clearly shown to be due to a single base change conferring an amino acid $\mathrm{F} \rightarrow \mathrm{Y}$ substitution in the clamp domain of the RPA190 subunit of RNA polymerase 1 (Randall et al. 2014). Strain 968-J2 of P. tabacina is sensitive to metalaxyl, while strain $968-$ S26 is insensitive. Comparisons of the gene encoding the RPA190 subunit of RNA polymerase I revealed the presence of $\mathrm{F}$ in both strains at the position of the substitution causing metalaxyl insensitivity in some strains of Phytophthora infestans. Therefore, a substitution at this site is not the basis for metalaxyl insensitivity in strain 968-S26. Analyses of read depth did not indicate that copy number variation in genes encoding RNA polymerase I was a determinant of (in)sensitivity to metalaxyl.

\section{Gene contexts.}

The distance between genes provides a measure of local gene density and an overview of global genome architecture. Whole genome analysis revealed regions in which gene density is high and repeat content is low; these regions have median intergenic distances within conserved gene blocks of 669 (968-J2) and 627 bp (968-S26). In regions between conserved blocks, the median intergenic distances are greater, around 1.3 (968-J2) and around $1.2 \mathrm{~kb}(968-\mathrm{S} 26)$. The gene-rich component comprises 7,456 (about $68 \%$ ) of protein-coding genes. Similar distribution of genes within and between conserved blocks were observed in $P$. ramorum (median $633 \mathrm{bp}$ and $1.5 \mathrm{~kb}$, genome size $65 \mathrm{Mb}$ ) (Haas et al. 2009). The distribution of RxLR domains across the genome was determined using the Bedtools suite, subcommand window (Supplementary Table S11). Approximately $43 \%$ of the RxLR domains in the two $P$. tabacina strains (56 of 122 in $968-\mathrm{J} 2$ and 47 of 117 in 968-S26) are located in gene-rich regions while about $57 \%$ are located in gene-sparse regions (67 of 122 in 968-J2 and 68 of 117 in 968S26) (Fig. 5), similar to that observed in Phytophthora infestans (Haas et al. 2009). Calculation of the mean gene density per scaffold based on the number of genes per kilobase reiterated that RxLR-containing proteins are located in gene-sparse regions (approximately 0.2 genes per kilobase compared with approximately 0.4 for the whole genome) (Table 7 ).

\section{Synteny.}

Extensive colinearity of syntelogs was observed between the two P. tabacina genomes. A total of $56 \%$ (about $36 \mathrm{Mb}$ ) of the 968-J2 genome could be shown to be syntenic to $65 \%$ (about $36 \mathrm{Mb}$ ) of the 968-S26 genome (Supplementary Table $\mathrm{S} 12$ ); $38 \%$ (about $24 \mathrm{Mb}$ ) of the genome could not be used for the calculation of synteny in CoGe, due to low gene density (CoGe requires at least five gene models per scaffold). The remaining $6 \%$ (about $3 \mathrm{Mb}$ ) was potentially not syntenic. The 25 largest scaffolds of $968-\mathrm{J} 2$ aligned to 111 scaffolds of 968 S26 (Fig. 6). There were only two instances in which a single 968-S26 scaffold linked to two 968-J2 scaffolds; however, since these links occurred at the edge of the scaffolds, it is unlikely they were due to mis-assembly and may, instead, indicate that the two scaffolds belong to the same DNA fragment. Since the two $P$. tabacina strains are highly syntenic, interspecific comparisons utilized only strain 968-J2 as the reference sequence. Aligning strain 968-J2 to the H. arabidopsidis isolate Emoy2 revealed that approximately $36 \%$ of the two assemblies were syntenic. In addition, 45 to $50 \%$ of the $968-\mathrm{J} 2$ genome was syntenic to 52 to $69 \%$ of three Phytophthora genomes (Fig. 6). At the microsyntenic level, blocks of synteny between $P$. tabacina and these other oomycetes show inversions and instances of expansion in intergenic regions (Supplementary Fig. S4). However, extensive rearrangements of the gene-rich genomic regions do not appear to have occurred during the evolution of these highly variable pathogens. Twenty-eight candidate RxLR-encoding genes (13 in 968-J2 and 15 in 968-S26) were located in regions that were syntenic across all five species. While many of these candidate effectors are located in gene-rich regions, about $20 \%$ are in highly variable regions of the $P$. tabacina genome.

\section{Mitochondrial genome.}

The mitochondrial genome was assembled with SeqMan NGen using a contig similar to a Phytophthora ramorum mitochondrial sequence as the seed template. The reads provided $9,142 \times$ and $6,824 \times$ coverage and were assembled into single circular genomes of 43,225 and 42,990 bp with GC contents of 22.4 and 22.35 for $968-\mathrm{J} 2$ and $968-$ S26, respectively (Supplementary Fig. S6) (GenBank accessions KT893455 and KT893456). The two genomes were highly similar differing only by seven SNP and three small indels as well as the copy number of a 128-bp repeat (Supplementary Table S13; Supplementary Fig. S7). The genome organization was highly colinear with several Phytophthora spp. (has the same gene order as Phytophthora ramorum and Phytophthora sojae) and exhibits some conservation of gene order in comparisons with Pythium ultimum and the more distantly related Saprolegnia ferax (Fig. 7). Coding regions represent $90.7 \%$ of the genome, with $15 \%$ of this total representing hypothetical coding regions. A total of 35 known genes (encoding 18 respiratory chain proteins, 16 ribosomal proteins, and an import protein, ymf16 of the $\sec$ Y-independent pathway), the $r n l$ and $r n s$, a total of 25 tRNA genes encoding for 19 amino acids were present in the mitochondrial genome. In addition, there were four hypothetical proteins (ymf98, ymf99, ymf100, and ymf101) in common with other oomycete mitochondrial genomes (Grayburn et al. 2004; Lévesque et al. 2010; Martin et al. 2007) and seven putative ORF located between the rns and cox 2 genes that are unique to $P$. tabacina (orf66, orf172, orf182, orf269a, orf $269 b$, orf290, and orf374). BLAST queries to GenBank identified no significant sequence similarities to orf66, orf182, orf $269 \mathrm{~b}$ and orf 290; however, there was moderate sequence similarity with putative ORF in mitochondrial genomes of Phytophthora spp. In $\operatorname{orf172}$, bases 134 to 518 are $85 \%$ identical to the $3^{\prime}$ end of orf1 $12 a$ and the $5^{\prime}$ end of orf112b of Phytophthora sojae (DQ832717). In orf269a, bases 323 to 743 are $85 \%$ identical to the spacer and $5^{\prime}$ end of orf116, while bases 59 to 320 are $89 \%$ identical to part of orf101 from Phytophthora sojae. In orf374,

Table 7. Gene density of effector containing scaffolds in the Peronospora tabacina assemblies

\begin{tabular}{lccccc}
\hline & \multicolumn{2}{c}{$\mathbf{9 6 8 - J 2}$} & & \multicolumn{2}{c}{$\mathbf{9 6 8 - S 2 6}$} \\
\cline { 2 - 3 } \cline { 5 - 6 } & Whole genome & RxLR-containing scaffolds & & Whole genome & RxLR-containing scaffolds \\
\hline Mean number of genes per scaffold & 6.58 & 15.21 & 7.20 & 14.81 \\
Mean scaffold size & 32,974 & 02,456 & 33,508 & 72,266 \\
Mean gene density* & 0.41 & 0.19 & 0.36 & 0.21 \\
\hline
\end{tabular}

\footnotetext{
* Average number of genes per $1 \mathrm{~kb}$ per scaffold.
} 
bases 6 to 419 have $76 \%$ identity to the $5^{\prime}$ end of orf 80 and the last 91 bp of orf99 from Phytophthora infestans (AY898627).

\section{DISCUSSION}

In this study, we describe a draft genome sequence for $P$. tabacina that is comparable to those of other sequenced oomycete species. This assembly is the result of combining the outputs of two different assembly programs to obtain a complete genome assembly. De novo nuclear and mitochondrial genomic assemblies were generated and characterized for two strains of $P$. tabacina (968-J2 and 968-S26). Both nuclear assemblies were shown to be comprehensive, using CEGMA. The colinearity of the two assemblies and their extensive synteny with other oomycete species indicates the high accuracy of the assemblies. Due to the great similarity between strains, the assembly of strain 968-J2 was selected as the reference for downstream interspecific comparisons.

The $P$. tabacina genome was estimated to be approximately $68 \mathrm{Mb}$, using both kmer and read coverage analyses, making it the smallest downy mildew genome published to date. Arabidopsis downy mildew (Hyaloperonospora arabidopsidis) isolate Emoy2, revealed an estimated genome size of $100 \mathrm{Mb}$ (Baxter et al. 2010). Genome sizes of 31 downy mildews have previously been estimated by Fuelgen staining (Voglmayr and Greilhuber 1998). Our estimation for P. tabacina of $68 \mathrm{Mb}$ falls within the size range of their genome estimates of 22 different Peronospora spp. (46 to $140 \mathrm{Mb}$ ). Of the two downy mildews for which draft genome or transcriptome assemblies are available, Pseudoperonospora cubensis (causing downy mildew of cucumber) has a predicted genome size $88 \mathrm{Mb}$ and the sizes of five isolates of Bremia lactucae (causing lettuce downy mildew) was estimated to be between 70 and $145 \mathrm{Mb}$ (Voglmayr and Greilhuber 1998; Wong 2011).

The genome size of $P$. tabacina is similar to that of Phytophthora ramorum (65 $\mathrm{Mb}, 28 \%$ repeats) and Phytophthora capsici (64 Mb, 19\% repeats), the pathogens causing sudden oak death and blight and fruit rot of vegetable crops, respectively (Lamour et al. 2012; Tyler et al. 2006). The amount of repetitive DNA elements identified in P. tabacina is proportional to the estimated genome size; it also has a low number of repetitive DNA elements, approximately $24 \%$ comparable to these two Phytophthora spp. Comparisons between Phytophthora infestans, Phytophthora ramorum, and Phytophthora sojae revealed that their differences in genome size are due to the proliferation of repetitive DNA sequences (Haas et al. 2009). The most abundant repetitive sequences identified in $P$. tabacina are long-terminal retrotransposons (Gypsy-like and Copia-like elements); while both strains had similar overall number of repeat sequences and families of repeats, the Gypsy family seems to have been active more recently in strain 968-S26 compared with 968-J2. The biological significance of this is unclear, since both strains are contemporary, being collected from the same field in Forchheim, Germany, in 2007 but have differences in virulence (Spring et al. 2013).

It is unclear whether sexual reproduction in P. tabacina is predominantly homothallic or heterothallic. Our sequence data indicate that there have recently been both outcrossing and selfing events in the ancestries of both strains. The genome assemblies revealed high levels of heterozygosity in each strain; more than $30 \%$ of the SNP that distinguished the two strains were heterozygous within a strain. This has consequences as to the veracity of the sequence of each strain. The two haplotypes are merged in over $80 \%$ of the assembly; therefore, the single linear nucleotide sequence is the consensus of the two haplotypes. As judged by read depth for $10 \%$ of the genome, each haplotype has been assembled independently, probably consisting of more variable regions of the genome. Consequently, functional studies of individual genes should utilize the raw reads and individual alleles generated from single haplotypes or sequences from polymerase chain reaction (PCR) products.

The overall number of genes predicted in $P$. tabacina is similar to those of other oomycetes; however, as expected, there was greater overlap in gene repertoires with other downy


Fig. 5. The genome architecture of Peronospora tabacina $968-\mathrm{J} 2$ and $968-\mathrm{S} 26$ plotted by $5^{\prime}$ and $3^{\prime}$ intergenic distances. The scale bar represents the number of genes in each bin, shown as a color-coded heat map. Genes in gene-rich regions are in red while genes in gene-poor regions are in blue. The genomic context for candidate RxLR effector genes (only possible for 61 of 122 for 968-J2 and 63 of 117 for 968-S26) were overlaid onto the heat map and are represented by circles. 
mildews than with Phytophthora spp. Approximately 18,000 protein-coding genes were predicted in each strain. Gene content, number, and length between the two strains were very similar; clustering based on sequence similarity led to the grouping of about 16,752 protein-coding gene groups into approximately 3,850 gene families. Clustering of predicted gene models across published oomyete species (Phytophthora infestans, Phytophthora ramorum, Phytophthora sojae, and $H$. arabidopsidis) revealed that 7,742 genes were shared between all five species; 3,473 were particular to these two downy mildew species. Gene ontology $(\mathrm{GO})$ analysis revealed that the genes unique to the downy mildews were enriched for RNA-dependent DNA replication (GO:0006278), RNAdirected DNA polymerase (GO:0003964), RNA binding (GO: 0003723), and cAMP binding (GO:0030552). Within the downy mildews, 5,693 were shared while 2,665 were unique to $P$. tabacina. Consistent with the lack of zoospores, the majority of genes encoding flagellar proteins were not observed by BLAST analysis with homologous sequences from Phytophthora spp. (Supplementary Table S14). Ten of the largest annotated protein families are not involved in pathogenesis-related processes.
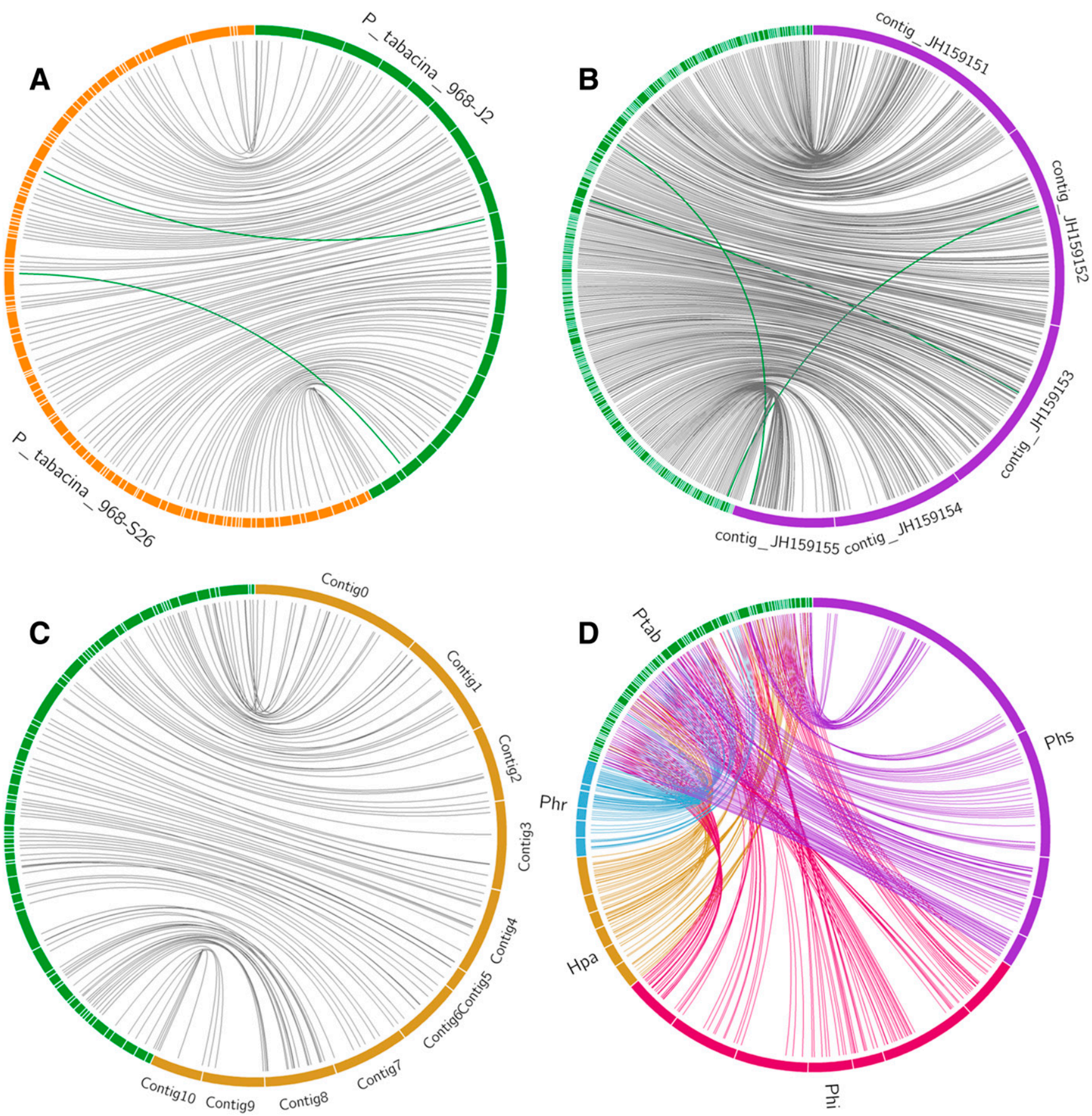

Fig. 6. Syntenic comparisons between Peronospora tabacina and published oomycete genomes. A, The 25 largest scaffolds in $P$. tabacina $968-\mathrm{J} 2$ to the 111 scaffolds of the 968-S26 genome. B, The five largest Phytophthora sojae scaffolds to the 286 scaffolds of P. tabacina 968-J2. C, The 10 largest Hyaloperonospora arabidopsidis scaffolds to the 74 scaffolds of P. tabacina 968-J2. D, The 89 scaffolds of P. tabacina 968-J2 (Ptab), Phytophthora infestans (Phi) (supercont1.4, supercont1.2, supercont1.7, supercont1.3, supercont1.6, supercont1.10, supercont1.1), Phytophthora ramorum (Phr) (scaffold_2, scaffold_3, scaffold_10, scaffold_5, scaffold_9, scaffold_1), and H. arabidopsidis (Hpa) (Contig1, Contig3, Contig4, Contig8, Contig7, Contig0) anchored to the five biggest Phytophthora sojae scaffolds (Phs) (contig_JH159151, contig_JH159152, contig_JH159153, contig_JH159154, contig_JH159155) to show colinearity between these species. 
The P. tabacina genome contains a large number of genes encoding proteins that are potentially involved in pathogenicity. Several protease inhibitors appear to be overrepresented in P. tabacina relative to their prevalence in Phytophthora spp. (Phytophthora infestans, Phytophthora ramorum, and Phytophthora sojae), while several families encoding host-targeted degradative enzymes (proteinases, cell wall-degrading enzymes) as well as elicitins are reduced (Table 3 ). Underrepresented proteins in the $P$. tabacina genome also included pectate lyases and phospholipases, these have been shown to target the host cell wall and elicit host defenses in P. capsici and bacteria (Collmer and Keen 1986; Jia et al. 2009). P. tabacina may have lost many of these enzymes during adaptation to a biotrophic lifestyle. This deficiency of hydrolytic enzymes was also observed in $H$. arabidopsidis (Baxter et al. 2010). Similarly, gene families encoding necrosis and ethylene inducing (Nep1)-like proteins (NLP) are also significantly reduced in P. tabacina. While NLP in Phytophthora and Pythium spp. trigger plant cell death and defense responses (Gijzen and Nürnberger 2006), the function of NLP in downy mildews is less clear; these proteins

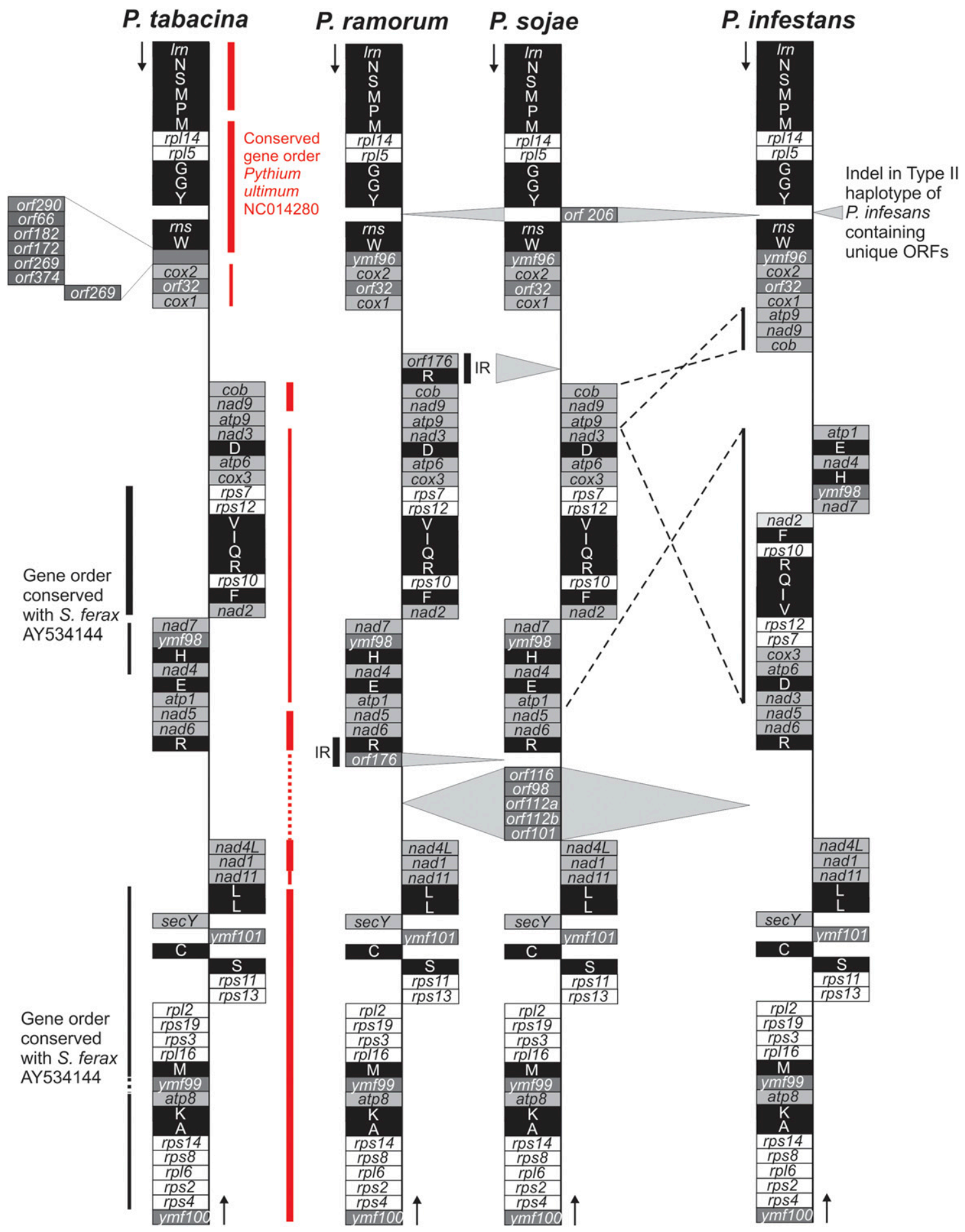

Fig. 7. The genome organization of the Peronospora tabacina mitochondrion and its similarity to the mitochondria of three Phytophthora spp. The shaded vertical line represents conservation of gene order with Pythium ultimum (NC014280), while the black vertical line represents comparison with Saprolegnia ferax (AY534144). Thicker lines indicate coding in the inverted repeat. 
may not elicit plant cell death and may have evolved functions that differ from those in hemibiotropic and necrotrophic oomycetes (Oome and Van den Ackerveken 2014; Oome et al. 2014; Seidl et al. 2015).

The largest and most diverse classes of pathogenicity-related proteins in $P$. tabacina contain an RxLR motif at their $\mathrm{N}$ termini that is common in secreted effector proteins of many oomycetes (Lamour et al. 2007). The P. tabacina genomes were mined using two different bioinformatics approaches, and both strains were shown to possess similar numbers of candidate RxLR effector encoding genes (122 in 968-J2 and 117 in 968-S26). This number is similar to that identified in $H$. arabidopsidis (Baxter et al. 2010) but less than that predicted in several Phytophthora spp. (350 to 563) (Haas et al. 2009; Kamoun 2006). The WY domain that is common in candidate effectors of other oomycetes (Boutemy et al. 2011; Win et al. 2012) was detected in a quarter of RxLR-containing proteins. The WY domain was also detected in predicted secreted proteins that lacked an RxLR motif. A total of $70 \%$ of the genes encoding RxLR effectors are orthologous between the two strains of $P$. tabacina; multiple orthologs appeared to be under diversifying selection with their $\mathrm{C}$ termini having a stronger signal. Each strain had six unique RxLR-encoding genes. Eighty-five effector clusters were private to $P$. tabacina compared with $H$. arabidopsidis and the three Phytophthora spp. Only one cluster was in common with $H$. arabidopsidis; this same cluster also had RxLR representatives from the three Phytophthora spp. While some clusters were common in pairwise comparisons with the three Phytophthora spp., most of the Phytophthora RxLR genes clustered together, forming the largest groups. The majority of effector-encoding genes are located in gene-sparse regions, although some are within generich regions as has been reported for Phytophthora infestans (Haas et al. 2009) Twenty-eight RxLR-encoding genes in the two $P$. tabacina strains are located in otherwise highly syntenic regions in other species, while most of these RxLR genes are located in gene-dense regions of the genome, $21 \%$ are in repeatrich regions.

The mitochondrial genome of $P$. tabacina is circular in orientation and does not have an inverted repeat that is present in some other oomycetes (Grayburn et al. 2004; Hudspeth et al. 1983; Lévesque et al. 2010; Mcnabb et al. 1987; O’Brien et al. 2014). At approximately $43 \mathrm{~kb}$, the size of the $P$. tabacina mitochondria is similar to what has been observed for Phytophthora spp. (approximately 38 to $43 \mathrm{~kb}$ ) and Pythium ultimum $(37.7 \mathrm{~kb}$ with only one arm of the inverted repeat, $59.7 \mathrm{~kb}$ total). The $P$. tabacina mitochondrial genome encodes the same standard suite of genes, including the putative ORF ymf16, ymf98, ymf99, ymf100, and ymf101, as has been reported for Phytophthora spp. (Martin et al. 2007) and Pythium ultimum (Lévesque et al. 2010) as well as Saprolegnia ferax (Grayburn et al. 2004), Achlya hypogyna, and Thraustotheca clavata (O'Brien et al. 2014). There are also an additional six putative ORF encoded between the rns and $\operatorname{cox} 2$ gene of unknown function that are unique to $P$. tabacina and share some level of sequence identity with unique putative ORF in Phytophthora infestans and Phytophthora sojae. The locations of these species-specific putative ORF were different in Phytophthora spp., as they were found upstream from the rns coding region or between nad6 and nad4L.

In general, the mitochondrial gene order in Phytophthora tabacina, Phytophthora ramorum, and Phytophthora sojae were identical with two differences caused by inversions when compared with Phytophthora infestans. These gene orders were also highly conserved in other oomycetes, although the overall genomes were not collinear due to rearrangements. For example, the trnL to ymf100 region is linked to the $r n l$ to $t r n P$ region in $P$. tabacina and Phytophthora spp., an organization that is highly conserved with the Peronosporomycete Pythium ultimum but not members of the Saprolegniomycetes (Grayburn et al. 2004; O'Brien et al. 2014). While the flanking trnM to $\operatorname{trn} W$ region in $P$. tabacina is also a conserved gene order in Pythium ultimum, it is located in a different region of the genome in this species. The gene orders trnL to ymf100 and rps7 to nad7 are also highly conserved in Saprolegnia ferax, as well as A. hypogyna and T. clavata (O'Brien et al. 2014).

There was limited intraspecific variation between the mitochondrial genomes of the two strains of $P$. tabacina, with a total of seven SNP (five in spacer regions, one synonymous, and one was nonsynonymous), three indels, and the number of copies of a 128-bp repeat estimated to be 3.23 in $968-\mathrm{J} 2$ and 1.47 in $968-$ S26. Based on this data, it should be possible to develop mitochondrial haplotype markers useful for following mitochondrial lineages in population studies, as has been done with Phytophthora ramorum, Phytophthora cinnamomi, and Phytophthora nicotianae (Mammella et al. 2013; Martin 2008; Martin and Coffey 2012).

The molecular mechanisms underlying $P$. tabacina-tobacco interactions are currently little-studied. The availability of genomic sequences will enable functional studies on the candidate effectors identified in this study in relation to the pathogenicity of $P$. tabacina. Tobacco is a model system for studying multiple plant-microbe interactions at the molecular level. This adds a biotrophic oomycete pathogen that can be studied on tobacco, including Nicotiana benthamiana, which is susceptible to $P$. tabacina. From a pathogenomics standpoint, this genome also provides a wealth of nuclear and mitochondrial markers for population genetic studies that will inform the epidemiology, variability, and potential control strategies for this destructive pathogen.

\section{MATERIALS AND METHODS}

\section{Strain origin and DNA isolation.}

Two strains of $P$. tabacina Adam, 968-J2 (sensitive to metalaxyl-M) and 968-S26 (tolerant to metalaxyl-M), were derived from a field isolate collected in 2007 at Forchheim, Germany, via single-spore infection of tobacco leaves, as described by Zipper et al. (2009). OS\#968, OS \#968-J2, and OS\#968-S26 are deposited as sporangia in the collection at the University of Hohenheim, Stuttgart, Germany. The strains were maintained under laboratory conditions through continuous infection of leaves of Nicotiana benthamiana. Fungicide sensitivity was determined repeatedly in leaf disk experiments, as described earlier (Rozynek and Spring 2001). Freshly emerged sporangiophores and sporangia were harvested from infected leaves, using a vacuum suction device (Thines et al. 2005). The pathogen material was disrupted with metal beads in a mixer mill $(9 \mathrm{~Hz}, 3 \mathrm{~min})$, and DNA was extracted following the protocol of Ristaino et al. (2001), with minor modifications.

\section{Genome sequencing and assembly.}

Paired-end libraries for sequencing were prepared from $1.5 \mu \mathrm{g}$ of total genomic DNA that had been fragmented using a Bioruptor sonicator (Diagenode, Denville, NJ, U.S.A.). The libraries were prepared using NEB (Ipswich, MA, U.S.A.) and Enzymatic (Beverly, MA, U.S.A.) reagents according to standard protocols. Size selection was performed after adaptor ligation for 450-bp fragments, using the PippinPrep (Sage Science, Beverly, MA, U.S.A.). The fragments were purified using 0.8× Agencourt Ampure beads XP (Beckman Coulter, Brea, CA, U.S.A.) and were eluted in $20 \mu$ of elution buffer. Mate-pair libraries were prepared using $4 \mu \mathrm{g}$ of total genomic 
DNA, using the Illumina Nextera mate pair sample preparation kit, according to the manufacturer's instructions. Selection of $5-$ and 12-kb fragments was performed using agarose gel electrophoresis and fragments were sonicated to approximately 750 bp, using the PippinPrep (Sage Science). Mate-pair libraries were enriched using 15 PCR cycles. Adaptors were obtained from Biooscientific (Austin, TX, U.S.A.). Library quality and quantity was confirmed using a Bioanalyzer (Agilent, Santa Clara, CA, U.S.A.) before sequencing. The sequences of the two strains of $P$. tabacina were generated on a Illumina MiSeq at the University of California Davis DNA Technologies Core. For each strain, paired-end and mate-pair sequencing runs were carried out using single pooled runs of 250 cycles each. For paired-end reads, adaptors were trimmed using Scythe, sequences were demultiplexed using allPrep-8.py and low-quality sequences were removed using Prinseq-lite (Schmieder and Edwards 2011) (parameters: trim_qual_right 28, trim_qual_type min, trim_qual_rule lt).

Mate-pair reads were demultiplexed and trimmed for quality (parameters: low_quality_cutoff 20, minLength 30) using the allPrep-8.py python script. Reads were processed, according to the Illumina processing guidelines of Nextera mate-pair data, using custom scripts. Since the mate-pair insert size was large (approximately $750 \mathrm{bp}$ ), reads with internal junction sequences and reads with no detectable junction sequences were maintained for downstream analyses. Read quality was monitored through the processing pipeline using FastQC.

In order to identify sequences from bacterial contaminants, paired-end and mate-pair reads were mapped back to a database of bacterial genome sequences that had been downloaded from the National Center for Biotechnology Information (NCBI), using BWA-MEM. Reads that did not map to bacteria were extracted as FastQ files from the BWA-MEM SAM output, using custom scripts for downstream processing.

The trimmed and filtered paired-end and mate-pair reads for each strain were assembled using ALLPATHS-LG (release 46402) (Butler et al. 2008) with ploidy $=2$, genome size $=$ $100 \mathrm{Mb}$, haploidify = true. A parallel assembly from the pairedend reads was generated using CLC Genomics Workbench v6.5; Qiagen, Boston). The completeness and continuity of the two assemblies was compared using the CEGMA (Parra et al. 2007) pipeline. Both assemblies were BLASTed to the NCBI nonredundant database to identify contigs and scaffolds of nonoomycete origin that were removed. The statistics for the ALLPATHS-LG assembly were superior, but the CEGMA scores were inferior to the CLC assembly; therefore, sequences unique to the CLC assembly were identified by mapping all paired-end reads to the ALLPATHS assembly and unmapped reads were extracted and reassembled in CLC. Redundant contigs were removed using BLAST to the ALLPaths-LG assembly, and contigs with $80 \%$ identity and $80 \%$ alignment were removed. The remaining contigs were then combined with the ALLPATHS assembly to generate a hybrid assembly, whose completeness was confirmed using CEGMA. The assembly was further reiteratively refined using the 'join contigs' feature of the CLC finishing module to join and align contigs. The assembly was then scaffolded using SSPACE, and positional information was generated by mapping reads using BWAMEM (Fig. 8).

The level of heterozygosity between the $P$. tabacina genomes was calculated by mapping reads from one isolate to the assembly of the other isolate, using BWA-MEM (reads of 968-J2 were mapped to the assembly of 968-S26 and vice versa). The SNP between the two genomes were counted using SAMTOOLs v0.1.18 (commands: sort, index, and mpileup and programs: bcftools and vcfutils.pl varFilter -D100). Heterozygous SNP were assigned based on having a major allele frequency between 0.4 and 0.6 , and homozygous SNP were assigned based on having a major allele frequency of either less than 0.4 , greater than 0.6 , or both. The haploid percentage of the genome was calculated based on read-depth analysis, was analyzed using custom scripts, and was visualized using $\mathrm{R}$.

To calculate the level of heterozygosity within each genome, in order to estimate when outcrossing may have occurred, reads of each strain were mapped back to each respective assembly, using BWA-MEM. The heterozygous SNP were assigned using the same method described above. These SNP were filtered to have a minimum read depth of 20 . A set of 11,310 and 10,707 predicted loci from isolate 968-J2 and 968-S26 were classified into homozygous and heterozygous states based on the presence of heterozygous SNP within each sequence, using the bedtools suite v2.17.0, subprogram 'intersect'. In addition, approximately $405 \mathrm{KOG}$ genes, shown to be orthologous using CEGMA, were analyzed to confirm the genome-wide analyses.

Genome size was independently estimated, using the read coverage provided by the Illumina reads and also in ALLPathsLG based on kmer analyses (Butler et al. 2008). To estimate the genome size, mean coverage for the Illumina reads was calculated for the haploid and diploid portions of the genome. Based on the proportion of the observed haploid percentage, a weighted mean coverage was determined. The maximum read depth cutoff used for the calculation was total read depth

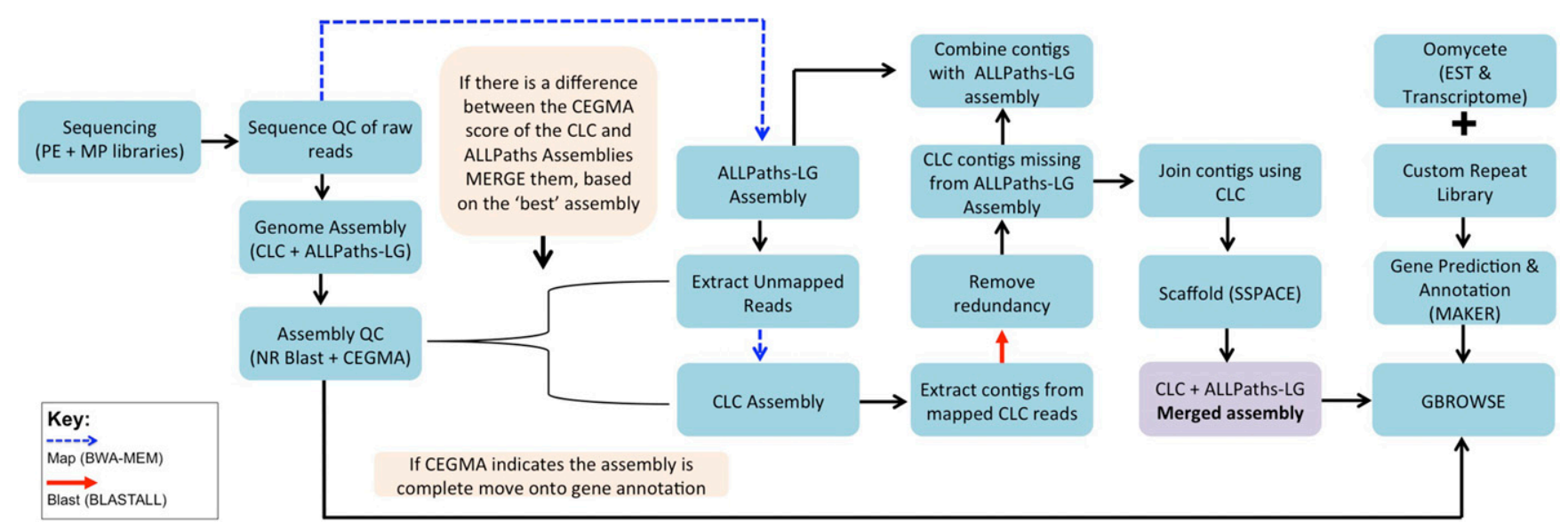

Fig. 8. The Peronospora tabacina assembly pipeline. 
mean $+2 \times$ standard deviation. The genome size was estimated by dividing the total length of the Illumina reads that mapped to the genome by the weighted mean, using the following formulas:

\section{Formula 1}

$$
\frac{\left(\mu_{A} \times N\right)+\left(\mu_{B} \times N^{\prime}\right)}{100}=\text { Weighted mean coverage }
$$

Where $\mu_{A}=$ mean read depth of the haploid portion of the genome, $N=$ percent of the genome representing one haplotype, $\mu_{B}=$ mean read depth of the nonhaploid portion of the genome, $N^{\prime}=$ percent of the genome representing merged haplotypes

Formula 2

Total number of mapped bases

Weighted mean coverage

For 968-J2

$$
\begin{gathered}
\frac{(37.82 \times 18)+(150.58 \times 82)}{100}=130.28 \\
\frac{8,801,330,937}{130.28}=67.6 \mathrm{Mb}
\end{gathered}
$$

For $968-\mathrm{S} 26$

$$
\begin{gathered}
\frac{(14.43 \times 17)+(71.33 \times 83)}{100}=61.66 \\
\frac{4,082,323,621}{61.66}=66.2 \mathrm{Mb}
\end{gathered}
$$

Kmer analyses in ALLPaths-LG resulted in an estimated genome size of $69 \mathrm{Mb}$. The close agreement of the genome size estimation methods suggests that the actual genome size of P. tabacina is approximately $68 \mathrm{Mb}$ (mean of the three estimates).

\section{Mitochondrial assembly and annotation.}

A contig from the initial genomic assembly was identified as mitochondrial due to sequence similarity with Phytophthora ramorum mitochondrial sequences (DQ832718). This was employed as a template for assembly with SeqMan NGen (version 4.1.2; DNASTAR, Madison, WI, U.S.A.). The resulting assembly was evaluated for uniformity and depth of coverage. Contigs were broken when gaps and low coverage or inconsistencies were observed, and the set of smaller contigs were reassembled using the small templated assembly option of SeqMan Ngen, to extend the ends of the contigs and the close gaps. ORF were predicted and annotated with DS Gene v1.5 (Accelrys, San Diego, CA, U.S.A.), using the universal genetic code, with confirmation of gene identities using BLAST analysis against published mitochondrial genome sequences from Pythium ultimum and Phytophthora spp. (Lévesque et al. 2010; Martin et al. 2007). TRNA coding regions were placed using tRNAscanSE v. 1.3.1 (Lowe and Eddy 1997). The number of copies of the 128-bp repeat in strain 968-J2 was confirmed to be more than two complete copies and the last repeat was confirmed incomplete by looking at individual reads. The final copy number was estimated by extracting the repeat region from the consensus contig and reassembling; the number of repeats was estimated to be the point where the average number of assembled reads was the same for the template with the repeat and without.

\section{Repeat identification and annotation.}

Generation of a repeat library. A custom repeat library for P. tabacina was constructed and annotated from strain $968-\mathrm{J} 2$ using the RepeatModeler v1.0.8 (Smit et al. 2013). Tandem repeats were identified using TRF finder v4.07b (Benson 1999) (parameters: match $=2$, mismatch $=7$, Delta $=7, \mathrm{PM}=80, \mathrm{PI}=10$, Minscore $=50$, MaxPeriod $=500$ ) and were converted into a gff3 file using a custom script.

The repeat content of the genome was determined by masking the repetitive elements of the assembly with RepeatMasker v3.3.0, using both the custom $P$. tabacina repeat library and the default repeat library (parameters: nolow, norna).

LTR analysis. LTR-RT elements were detected using ltrharvest (Ellinghaus et al. 2008) by screening for LTR oriented toward each other separated by 1 to $40 \mathrm{~kb}$. The predicted LTR-RT was annotated using ltrdigest (Steinbiss et al. 2009) to predict the polypurine-track and protein-binding site domains together with the internal protein-coding domains. The protein-coding domains were predicted using HMM profiles for protein domains known to be encoded by LTR-RT elements with sequence alignments from pFAM (Finn et al. 2014) (Supplementary Table S15). The LTR-RT were then clustered using VMatch based on similarity between their internal domains, as reported by Steinbiss et al. (2009). In parallel, all the predicted LTR-RT were aligned to the TREP repeat database (Wicker et al. 2002) using BLAST; each cluster of LTRRT was assigned to a repeat family and subfamily based on the BLAST hits as detailed in Wicker et al. (2007). After clustering and annotation, the age of each family of LTR-RT was calculated, using a modification of the method by Baucom et al. (2009) and Vitte et al. (2007). A multiple sequence alignment of all the $3^{\prime}$ and $5^{\prime}$ LTR within a cluster was done using Clustal-Omega 1.2.0 (Sievers et al. 2011). The divergence between the aligned $3^{\prime}$ and 5 ' LTR sequences of individual elements was estimated with BaseML from PAML (Yang 2007). The age of each element was calculated using the formula $T=d / 2 r$, where $d$ as the estimate of divergence and $\mathrm{r}$ is $1 \times 10^{-8}$ for host-encoded genes and was adjusted for the higher evolution rate of transposable elements compared with genes (Strasburg and Rieseberg 2008).

\section{Noncoding RNAs.}

The tRNAScan-SE v1.3.1 algorithm (Lowe and Eddy 1997) was applied with default parameters, using the eukaryotic model to the assembly to predict transfer RNAs. Ribosomal RNAs (rRNA) (5/8 s, 16/18 s, and 23/28 s) were predicted from the asssembly using RNAmmer v1.2 (Lagesen et al. 2007). Small nucleolar RNAs (snoRNAs) were predicted using snoscan v0.9b (Lowe and Eddy 1999), using the rRNA sequence file from RNAmmer as input.

\section{Annotation of nuclear genes.}

Genes were annotated using the MAKER v2.10 (Cantarel et al. 2008) annotation pipeline, with six iterations to provide a comprehensive set of predicted protein-coding sequences. cDNA data were collected from multiple sources in order to gather as many transcripts as possible. Complete transcriptome assemblies were downloaded from the Oomycete Transcriptomics database for Phytophthora infestans, Phytophthora sojae, and $H$. arabidopsidis and for Bremia lactucae BL24 from NCBI (accession number PRJNA77727). To complement the transcriptome data, the complete expressed sequence tag (EST) collection for the oomycete clade (taxonomy identification number 4762) of NCBI was downloaded, together with the protein collection from the same clade, to be used for the protein to genome alignments. Strain 968-J2 was first annotated using MAKER without an HMM and making predictions from mRNA sequence alignments (option -est2genome activated), using the default maker repeat libraries. A second iteration was done using the MAKER default repeat library and an HMM derived from Bremia lactucae (unpublished data). 
Custom HMM profiles were then generated based on the gene models from the two initial iterations of MAKER, using SNAP v2006-07-28 (Korf 2004) and Augustus v2.5.5 (Stanke et al. 2004). A third iteration of MAKER was run using the custom HMM and default repeat library. The initial three iterations were replicated using the custom repeat library built from the $968-\mathrm{J} 2$ genome. The same six iterations were performed again for strain 968-S26, using the HMM profiles and repeat library generated for $968-\mathrm{J} 2$. The six sets of gene models were merged by comparing their coordinates on the genomic sequence to obtain an inclusive set of gene models for each strain. This was reduced to a nonredundant set of gene models by eliminating predictions that had the same sequence, structure, and location. The predicted gene models were then filtered by comparing their structures with aligned EST and proteins. Gene models with either no support, matched predicted repeat elements, or both were removed. The remaining nonredundant filtered gene models were functionally annotated using Interproscan v5 (Jones et al. 2014) and were clustered into gene families using OrthoMCL v2.0.7 (Fischer et al. 2011). Blast2GO (Conesa et al. 2005) enrichment analyses were undertaken on gene models that were specific to the downy mildew clade. Information on clustered gene model was extracted from the outputs from OrthoMCL and Interproscan, using custom scripts. The gene models that overlapped were grouped into loci. The nonredundant gene model and loci datasets were filtered based on presence of transcriptome evidence and lack of predicted repeat elements within exons to obtain sets of reliable predicted protein coding and genic sequences.

Calculation of overrepresented and underrepresented gene families. A chi-square test was used to analyze changes in gene content of the two $P$. tabacina strains relative to the three Phytophtora spp. Two different methods were used. In the first method, each individual cluster was analyzed by using the total number of proteins annotated for all the genomes. In the second method, gene model clusters were collapsed based on their functional annotation. For both these methods, expected values were calculated by taking the mean number of genes and applying a standard factor, which was calculated based on the total number of clustered genes observed. To determine the level of significance, Bonferroni corrected $P$ values were used with a threshold of 0.05 .

\section{Identification of putative secreted proteins.}

The assemblies were translated to all possible ORF that encoded 80 or more aa from both strands of the genomic DNA sequence. SignalP v4.0 (Petersen et al. 2011) was used to identify an amino terminal signal peptide. Proteins showing a D score $\geq 0.45$ with no predicted transmembrane domains (SignalP-noTM) were considered to be within the P. tabacina secretome. The two secretomes were functionally annotated using Interproscan v5 (Jones et al. 2014) and were clustered into gene families using OrthoMCL v2.0.7 (Fischer et al. 2011).

Candidate RxLR effectors were selected from the secretome using custom Perl scripts. Secreted ORF with an RxLR motif downstream of the signal peptide cleavage site that possess a SignalP v4.0 cleavage site within the first 50 aa and an amino acid length $\geq 50$ from the end of the RxLR motif were selected as candidate RxLR effectors. RxLR effectors were first identified in the $P$. tabacina secretome with the hmmsearch program (HMMer v3.1) (Eddy 1998; Eddy 2011), using a published HMM profile that was generated from alignments of RxLREER-containing genes from Phytophthora infestans (Whisson et al. 2007). The candidate $P$. tabacina RXLR sequences that were identified using the Whisson et al. (2007) HMM were then trimmed to include the 5 to 10 aa before and after the
RXLR/RXLR-EER motifs and were aligned using the web version of Clustal Omega. The alignment was used as input in the hmmbuild program (Eddy 2011) to generate a $P$. tabacinaspecific RxLR HMM profile, and the secretome was searched using the hmmsearch program (Eddy 2011). RxLR sequencess were also identified using string searches for the $[\mathrm{G}, \mathrm{Q}, \mathrm{R}] \mathrm{xLR}$ motif within the secretome, 30 to 100 residues from the cleavage site of the predicted signal peptide. Redundant protein sequences due to translation of overlapping ORF and potential sequence duplication resulting from assembly artifacts were identified by BLASTP searches and were removed. Sequences showing $100 \%$ identity with an $E$ value of $<1 \mathrm{e}^{-10}$ were deemed redundant. Conserved domains were identified within the candidate RxLR effector protein sequences using Batch CDsearch (Marchler-Bauer et al. 2011). Predicted RxLR proteins identified in $P$. tabacina were clustered with RxLR proteins identified in the published genomes of $H$. arabidopsidis, Phytophthora infestans, Phytophthora ramorum, and Phytophthora sojae, using OrthoMCL v2.0.7. The genomic environment of the RxLR regions were classified as either being in gene-rich or gene-sparse regions of the genome, based on the percentage of predicted gene models relative to repetitive elements present, using the subcommand 'window' in bedtools suite v2.17.0. For predicted gene models, the $5^{\prime}$ and $3^{\prime}$ intergenic distances were used to determine the amount of overlap (parameters: 968-J2 -1 2800, -r 2700, -c; 968-S26 -1 2300, -r 2300, -c). For repeat regions, default parameters were used.

To identify WY domain-containing proteins, a HMM profile was built using HMMer v3.1 (Eddy 2011) from WY domaincontaining amino acid sequences of three related Phytophthora species (Boutemy et al. 2011). The HMM was used to search the $P$. tabacina translated ORF sequences (minimum ORF length $80 \mathrm{aa}$ ) and putative secretome. Hits with a positive HMM score were considered to be putative WY domain effectors, using inclusion criteria from Boutemy et al. (2011). The full amino-acid sequences of the candidate WY effectors were aligned using Clustal Omega, and Clustal phylogeny was used to construct a neighbor joining tree. This tree was annotated using Interactive Tree of Life.

CRN were detected from the $P$. tabacina genome using HMM profiles that were built using HMMer v3.1 (Eddy 2011) from CRN-containing amino-acid sequences obtained from Haas et al. (2009). These sequences were aligned using Clustal Omega. The HMM was used to search the translated ORF sequences (minimum ORF length, 80 aa) and the secretome using the hmmsearch function. Hits with a positive HMM score were considered to be putative CRN.

\section{Positive selection analysis of candidate RXLR and WY domain-containing effectors.}

Identification of alleles of $P$. tabacina candidate RxLR effectors between strains 968-J2 (122 candidates) and 968-S26 (117 candidates) were through bidirectional BLASTP searches ( $\mathrm{E}$ value cutoff $<\mathrm{e}^{-5}$ ) of protein sequences. A total of 86 sequences returned a hit, using a reciprocal blast search. These sequences were filtered based on $\geq 80 \%$ alignment, no or few gaps across the aligned sequences, and $\geq 95 \%$ identity. The coding DNA sequences for these allelic pairs were extracted based on location data. The rates of nonsynonymous nucleotide substitutions per nonsynonymous site $\left(D_{n}\right)$ and the rates of synonymous nucleotide substitutions per synonymous site $\left(D_{s}\right)$ were calculated across pairwise distances of each of the P. tabacina RxLR allele pair, according to the methods of Nei and Gojobori (1986) using PAL2NAL (Suyama et al. 2006). Protein sequences were aligned using Clustal Omega v1.2.0 (default parameters). $D_{n}$ and $D_{s}$ substitution rates were calculated using full-length sequences. In addition, the protein and 
nucleotide sequences were split in half to calculate the rates of substitution both at the $\mathrm{N}$-terminus and the $\mathrm{C}$-terminus. The sequences were split based on the amino-acid alignment using custom scripts, in which the $\mathrm{N}$-terminal regions represent the start codon to the middle of sequence and the C-terminal regions represents the middle to the end of the sequence.

For WY domain-containing proteins, allelic pairs (63 candidates in 968-J2 and 60 candidates in 968-S26) were identified through bidirectional BLASTP searches (E value cutoff $<\mathrm{e}^{-5}$ ) of the protein sequences. A total of 29 sequences returned a hit using reciprocal blast searches. The $\mathrm{D}_{\mathrm{n}}$ and $\mathrm{D}_{\mathrm{s}}$ substitution rates were calculated as above.

\section{Visualization of whole-genome architecture.}

The analysis of localized gene density was performed by plotting the abundance of genes according to $5^{\prime}$ and $3^{\prime}$ flanking intergenic regions. Calculation of intergenic distance and 2-dimensional data binning were based on the R script of Saunders et al. (2014), modified to utilize data of overlapping genes.

\section{Synteny analysis.}

The annotated genomic sequences for the two $P$. tabacina strains were uploaded into CoGe (Lyons and Freeling 2008), the online comparative genomics platform. The SynMap function was used to detect syntenic regions between the two strains and to determine levels of colinearity against published oomycete genomes (H. arabidopsidis, Phytophthora infestans, Phytophthora ramorum, Phytophthora sojae). The CoGe output (final syntenic gene set) was parsed using custom scripts and the syntenic blocks were visualized using Circos v0.66 (macro-synteny) (Krzywinski et al. 2009) and the multi Genome Synteny Viewer (microsynteny) (Revanna et al. 2012). Syntenic ranges were extracted using custom scripts based on the coordinates of the first and last gene in each syntenic block for all pairwise comparisons. The location of these regions enabled the identification of blocks of synteny present across a panel of oomycetes and also allowed for the downstream assessment of genomic features within syntenic regions of these species.

\section{Data access.}

Genomic sequences have been deposited in the GenBank database under accession number PRJNA285243. The mitochondrial genome is deposited under GenBank accession numbers KT893455 and KT893456). Genome browsers have been set up for both strains on the University of California Davis website. The genome assemblies and annotation files (GFF3) will also be available for download from the genome browser URLs (addresses are published in the AuthorRecommended Internet Resource section).

\section{ACKNOWLEDGMENTS}

The authors would like to thank T. Ho and K. Cox, undergraduate students in the Michelmore Lab, for bioinformatics contributions to this project. We also thank R. Zipper, Institute of Botany, University of Hohenheim, for maintainance of the pathogen strains and for providing the sporangial material. This project was funded by an endowment from Novozymes Inc. to R. W. Michelmore.

\section{LITERATURE CITED}

Adhikari, B. N., Hamilton, J. P., Zerillo, M. M., Tisserat, N., Lévesque, C. A., and Buell, C. R. 2013. Comparative genomics reveals insight into virulence strategies of plant pathogenic oomycetes. PLoS One 8:e75072.

Bartnicki-Garcia, S. 1968. Cell wall chemistry, morphogenesis, and taxonomy of fungi. Annu. Rev. Microbiol. 22:87-108.

Baucom, R. S., Estill, J. C., Leebens-Mack, J., and Bennetzen, J. L. 2009. Natural selection on gene function drives the evolution of LTR retrotransposon families in the rice genome. Genome Res. 19:243-254.
Baxter, L., Tripathy, S., Ishaque, N., Boot, N., Cabral, A., Kemen, E., Thines, M., Ah-Fong, A., Anderson, R., Badejoko, W., Bittner-Eddy, P., Boore, J. L., Chibucos, M. C., Coates, M., Dehal, P., Delehaunty, K., Dong, S., Downton, P., Dumas, B., Fabro, G., Fronick, C., Fuerstenberg, S. I., Fulton, L., Gaulin, E., Govers, F., Hughes, L., Humphray, S., Jiang, R. H. Y., Judelson, H., Kamoun, S., Kyung, K., Meijer, H., Minx, P. Morris, P., Nelson, J., Phuntumart, V., Qutob, D., Rehmany, A., RougonCardoso, A., Ryden, P., Torto-Alalibo, T., Studholme, D., Wang, Y., Win, J., Wood, J., Clifton, S. W., Rogers, J., Van den Ackerveken, G., Jones, J. D. G., McDowell, J. M., Beynon, J., and Tyler, B. M. 2010. Signatures of adaptation to obligate biotrophy in the Hyaloperonospora arabidopsidis genome. Science 330:1549-1551.

Benson, G. 1999. Tandem repeats finder: A program to analyze DNA sequences. Nucleic Acids Res. 27:573-580.

Boetzer, M., Henkel, C. V., Jansen, H. J., Butler, D., and Pirovano, W. 2011. Scaffolding pre-assembled contigs using SSPACE. Bioinformatics 27: 578-579.

Boutemy, L. S., King, S. R. F., Win, J., Hughes, R. K., Clarke, T. A., Blumenschein, T. M., Kamoun, S., and Banfield, M. J. 2011. Structures of Phytophthora RXLR effector proteins: A conserved but adaptable fold underpins functional diversity. J. Biol. Chem. 286:35834-35842.

Bruck, R. I., Gooding, G. V., and Main, C. E. 1982. Evidence for resistance to metalaxyl in isolates of Peronospora tabacina. Plant Dis. 66:44-45.

Burruano, S. 2000. The life-cycle of Plasmopara viticola, cause of downy mildew of vine. Mycologist 14:179-182.

Butler, J., Maccallum, I., Kleber, M., Shlyakhter, I. A., Belmonte, M. K., Lander, E. S., Nusbaum, C., and Jaffe, D. B. 2008. ALLPATHS : De novo assembly of whole-genome shotgun microreads. Genome Res. 18:810-820.

Cantarel, B. L., Korf, I., Robb, S. M. C., Parra, G., Ross, E., Moore, B., Holt, C., Sánchez Alvarado, A., and Yandell, M. 2008. MAKER: An easy-to-use annotation pipeline designed for emerging model organism genomes. Genome Res. 18:188-196.

Cohen, Y., and Coffey, M. 1986. Systemic fungicides and the control of oomycetes. Annu. Rev. Phytopathol. 24:311-338.

Collmer, A., and Keen, N. T. 1986. The Role of pectic enzymes in plant pathogenesis. Annu. Rev. Phytopathol. 24:383-409.

Conesa, A., Götz, S., García-Gómez, J. M., Terol, J., Talón, M., and Robles, M. 2005. Blast2GO: A universal tool for annotation, visualization and analysis in functional genomics research. Bioinformatics 21:3674-3676.

Davidse, L. C., Hofman, A. E., and Velthius, G. C. M. 1983. Specific interference of metalaxyl with endogenous RNA polymerase activity in isolated nucle from Phytophthora megasperma f . sp . medicaginis. Exp. Mycol. 7:344-361

Eddy, S. R. 1998. Profile hidden Markov models. Bioinformatics 14: 755-763.

Eddy, S. R. 2011. Accelerated profile HMM searches. PLOS Comput. Biol. 7:e1002195.

Ellinghaus, D., Kurtz, S., and Willhoeft, U. 2008. LTRharvest, an efficient and flexible software for de novo detection of LTR retrotransposons. BMC Bioinformatics 9:18.

Finn, R. D., Bateman, A., Clements, J., Coggill, P., Eberhardt, R. Y., Eddy, S. R., Heger, A., Hetherington, K., Holm, L., Mistry, J., Sonnhammer, E. L. L., Tate, J., and Punta, M. 2014. Pfam: The protein families database. Nucleic Acids Res. 42:D222-D230.

Fischer, S., Brunk, B. P., Chen, F., Gao, X., Harb, O. S., Iodice, J. B., Shanmugam, D., Roos, D. S., and Stoeckert, C. J. 2011. Using OrthoMCL to assign proteins to OrthoMCL-DB groups or to cluster proteomes into new ortholog groups. Pages 6.12.1-6.12.19 in: Current Protocols in Bioinformatics, John Wiley \& Sons, Inc., New York.

Gijzen, M., and Nürnberger, T. 2006. Nep1-like proteins from plant pathogens: Recruitment and diversification of the NPP1 domain across taxa. Phytochemistry 67:1800-1807.

Grayburn, W. S., Hudspeth, D. S. S., Gane, M. K., and Hudspeth, M. E. S. 2004. The mitochondrial genome of Saprolegnia ferax: Organization, gene content and nucleotide sequence. Mycologia 96:981-989.

Haas, B. J., Kamoun, S., Zody, M. C., Jiang, R. H. Y., Handsaker, R. E., Cano, L. M., Grabherr, M., Kodira, C. D., Raffaele, S., Torto-Alalibo, T., Bozkurt, T. O., Ah-Fong, A. M. V., Alvarado, L., Anderson, V. L., Armstrong, M. R., Avrova, A., Baxter, L., Beynon, J., Boevink, P. C., Bollmann, S. R., Bos, J. I. B., Bulone, V., Cai, G., Cakir, C., Carrington, J. C., Chawner, M., Conti, L., Costanzo, S., Ewan, R., Fahlgren, N., Fischbach, M. A., Fugelstad, J., Gilroy, E. M., Gnerre, S., Green, P. J., Grenville-Briggs, L. J., Griffith, J., Grünwald, N. J., Horn, K., Horner, N. R., Hu, C. H., Huitema, E., Jeong, D. H., Jones, A. M., Jones, J. D., Jones, R. W., Karlsson, E. K., Kunjeti, S. G., Lamour, K., Liu, Z., Ma, L., Maclean, D., Chibucos, M. C., McDonald, H., McWalters, J., Meijer, H. J., Morgan, W., Morris, P. F., Munro, C. A., O’Neill, K., OspinaGiraldo, M., Pinzón, A., Pritchard, L., Ramsahoye, B., Ren, Q., Restrepo, S., Roy, S., Sadanandom, A., Savidor, A., Schornack, S., 
Schwartz, D. C., Schumann, U. D., Schwessinger, B., Seyer, L., Sharpe, T., Silvar, C., Song, J., Studholme, D. J., Sykes, S., Thines, M., van de Vondervoort, P. J., Phuntumart, V., Wawra, S., Weide, R., Win, J., Young, C., Zhou, S., Fry, W., Meyers, B. C., van West, P., Ristaino, J., Govers, F., Birch, P. R., Whisson, S. C., Judelson, H. S., and Nusbaum, C. 2009. Genome sequence and analysis of the Irish potato famine pathogen Phytophthora infestans. Nature 461:393-398.

Heist, E., Nesmith, W., and Schardl, C. 2002. Interactions of Peronospora tabacina with roots of Nicotiana spp. in gnotobiotic associations. Phytopathology 92:400-405.

Hudspeth, M. E. S., Shumard, D. S., Bradford, C. J. R., and Grossman, L. I 1983. Organization of Achlya mtDNA: A population with two orientations and a large inverted repeat containing the rRNA genes. Proc. Natl. Acad. Sci. U.S.A. 80:142-146

Jia, Y. J., Feng, B. Z., Sun, W. X., and Zhang, X. G. 2009 Polygalacturonase, pectate lyase and pectin methylesterase activity in pathogenic strains of Phytophthora capsici incubated under different conditions. J. Phytopathol. 157:585-591.

Jiang, R. H. Y., Tripathy, S., Govers, F., and Tyler, B. M. 2008. RXLR effector reservoir in two Phytophthora species is dominated by a single rapidly evolving superfamily with more than 700 members. Proc. Natl. Acad. Sci. U.S.A. 105:4874-4879.

Jiang, R. H. Y., and Tyler, B. M. 2012. Mechanisms and evolution of virulence in oomycetes. Annu. Rev. Phytopathol. 50:295-318.

Jones, P., Binns, D., Chang, H.-Y., Fraser, M., Li, W., McAnulla, C., McWilliam, H., Maslen, J., Mitchell, A., Nuka, G., Pesseat, S., Quinn, A. F., Sangrador-Vegas, A., Scheremetjew, M., Yong, S.-Y., Lopez, R., and Hunter, S. 2014. InterProScan 5: Genome-scale protein function classification. Bioinformatics 30:1236-1240.

Kale, S. D., Gu, B., Capelluto, D. G. S. S., Dou, D., Feldman, E., Rumore, A., Arredondo, F. D., Hanlon, R., Fudal, I., Rouxel, T., Lawrence, C. B., Shan, W., and Tyler, B. M. 2010. External lipid PI3P mediates entry of eukaryotic pathogen effectors into plant and animal host cells. Cell 142: 284-295.

Kamoun, S. 2006. A catalogue of the effector secretome of plant pathogenic oomycetes. Annu. Rev. Phytopathol. 44:41-60.

Kemen, E., Gardiner, A., Schultz-Larsen, T., Kemen, A. C., Balmuth, A. L., Robert-Seilaniantz, A., Bailey, K., Holub, E., Studholme, D. J., Maclean, D., and Jones, J. D. G. 2011. Gene gain and loss during evolution of obligate parasitism in the white rust pathogen of Arabidopsis thaliana. PLoS Biol. 9:e1001094.

Korf, I. 2004. Gene finding in novel genomes. BMC Bioinformatics 5:59.

Krzywinski, M., Schein, J., Birol, I., Connors, J., Gascoyne, R., Horsman, D., Jones, S. J., and Marra, M. A. 2009. Circos: An information aesthetic for comparative genomics. Genome Res. 19:1639-1645.

Lagesen, K., Hallin, P., Rødland, E. A., Staerfeldt, H.-H., Rognes, T., and Ussery, D. W. 2007. RNAmmer: Consistent and rapid annotation of ribosomal RNA genes. Nucleic Acids Res. 35:3100-3108.

Lamour, K. H., Mudge, J., Gobena, D., Hurtado-Gonzales, O. P., Schmutz, J., Kuo, A., Miller, N. A., Rice, B. J., Raffaele, S., Cano, L. M., Bharti, A. K., Donahoo, R. S., Finley, S., Huitema, E., Hulvey, J., Platt, D., Salamov, A., Savidor, A., Sharma, R., Stam, R., Storey, D., Thines, M., Win, J., Haas, B. J., Dinwiddie, D. L., Jenkins, J., Knight, J. R., Affourtit, J. P., Han, C. S., Chertkov, O., Lindquist, E. A., Detter, C., Grigoriev, I. V., Kamoun, S., and Kingsmore, S. F. 2012. Genome sequencing and mapping reveal loss of heterozygosity as a mechanism for rapid adaptation in the vegetable pathogen Phytophthora capsici. Mol. Plant-Microbe Interact. 25:1350-1360.

Lamour, K. H., Win, J., and Kamoun, S. 2007. Oomycete genomics: New insights and future directions. FEMS Microbiol. Lett. 274:1-8.

Lévesque, C. A., Brouwer, H., Cano, L., Hamilton, J. P., Holt, C., Huitema, E., Raffaele, S., Robideau, G. P., Thines, M., Win, J., Zerillo, M. M., Beakes, G. W., Boore, J. L., Busam, D., Dumas, B., Ferriera, S., Fuerstenberg, S. I., Gachon, C. M. M., Gaulin, E., Govers, F., GrenvilleBriggs, L., Horner, N., Hostetler, J., Jiang, R. H. Y., Johnson, J., Krajaejun, T., Lin, H., Meijer, H. J. G., Moore, B., Morris, P., Phuntmart, V., Puiu, D., Shetty, J., Stajich, J. E., Tripathy, S., Wawra, S., van West, P., Whitty, B. R., Coutinho, P. M., Henrissat, B., Martin, F., Thomas, P. D., Tyler, B. M., De Vries, R. P., Kamoun, S., Yandell, M., Tisserat, N., and Buell, C. R. 2010. Genome sequence of the necrotrophic plant pathogen Pythium ultimum reveals original pathogenicity mechanisms and effector repertoire. Genome Biol. 11:R73.

Li, H., Handsaker, B., Wysoker, A., Fennell, T., Ruan, J., Homer, N., Marth, G., Abecasis, G., Durbin, R., and 1000 Genome Project Data Processing Subgroup. 2009. The sequence alignment/map format and SAMtools. Bioinformatics 25:2078-2079.

Lowe, T. M., and Eddy, S. R. 1997. tRNAscan-SE: A program for improved detection of transfer RNA genes in genomic sequence. Nucleic Acids Res. 25:955-964.
Lowe, T. M., and Eddy, S. R. 1999. A computational screen for methylation guide snoRNAs in yeast. Science 283:1168-1171.

Lyons, E., and Freeling, M. 2008. How to usefully compare homologous plant genes and chromosomes as DNA sequences. Plant J. 53:661-673.

Mammella, M. A., Martin, F. N., Cacciola, S. O., Coffey, M. D., Faedda, R., and Schena, L., 2013. Analyses of the population structure in a global collection of Phytophthora nicotianae isolates inferred from mitochondrial and nuclear DNA sequences. Phytopathology 103:610-622.

Marchler-Bauer, A., Lu, S., Anderson, J. B., Chitsaz, F., Derbyshire, M. K., DeWeese-Scott, C., Fong, J. H., Geer, L. Y., Geer, R. C., Gonzales, N. R., Gwadz, M., Hurwitz, D. I., Jackson, J. D., Ke, Z., Lanczycki, C. J., Lu, F., Marchler, G. H., Mullokandov, M., Omelchenko, M. V., Robertson, C. L., Song, J. S., Thanki, N., Yamashita, R. A., Zhang, D., Zhang, N., Zheng, C., and Bryant, S. H. 2011. CDD: A conserved domain database for the functional annotation of proteins. Nucleic Acids Res. 39:D225-D229.

Martin, F. N. 2008. Mitochondrial haplotype determination in the oomycete plant pathogen Phytophthora ramorum. Curr. Genet. 54:23-34.

Martin, F. N., Bensasson, D., Tyler, B. M., and Boore, J. L. 2007. Mitochondrial genome sequences and comparative genomics of Phytophthora ramorum and P. sojae. Curr. Genet. 51:285-296.

Martin, F. N., and Coffey, M. D. 2012. Mitochondrial haplotype analysis for differentiation of isolates of Phytophthora cinnamomi. Phytopathology 102:229-239

McKeen, W. E. 1989. Blue Mold of Tobacco. First Edition. W. E. McKeen, ed. American Phytopathological Society, St. Paul, MN, U.S.A.

Mcnabb, S. A., Boyd, D. A., Belkhiri, A., Dick, M. W., and Klassen, G. R. 1987. An inverted repeat comprises more than three-quarters of the mitochondrial genome in two species of Pythium. Curr. Genet. 12:205-208.

Michelmore, R., and Wong, J. 2008. Classical and molecular genetics of Bremia lactucae, cause of lettuce downy mildew. Eur. J. Plant Pathol. 122:19-30.

Nei, M., and Gojobori, T. 1986. Simple methods for estimating the numbers of synonymous and nonsynonymous nucleotide substitutions. Mol. Biol. Evol. 3:418-426.

O'Brien, M. A., Misner, I., and Lane, C. E. 2014. Mitochondrial genome sequences and comparative genomics of Achlya hypogyna and Thraustotheca clavata. J. Eukaryot. Microbiol. 61:146-154.

Oome, S., Raaymakers, T. M., Cabral, A., Samwel, S., Böhm, H., Albert, I., Nürnberger, T., and Van den Ackerveken, G. 2014. Nep1-like proteins from three kingdoms of life act as a microbe-associated molecular pattern in Arabidopsis. Proc. Natl. Acad. Sci. U.S.A. 111:16955-16960.

Oome, S., and Van den Ackerveken, G. 2014. Comparative and functional analysis of the widely occurring family of Nep1-like proteins. Mol. Plant-Microbe Interact. 27:1081-1094.

Parra, G., Bradnam, K., and Korf, I. 2007. CEGMA: A pipeline to accurately annotate core genes in eukaryotic genomes. Bioinformatics 23:1061-1067.

Perumal, R., Nimmakayala, P., Erattaimuthu, S. R., No, E.-G., Reddy, U. K., Prom, L. K., Odvody, G. N., Luster, D. G., and Magill, C. W. 2008 Simple sequence repeat markers useful for sorghum downy mildew (Peronosclerospora sorghi) and related species. BMC Genet. 9:77.

Petersen, T. N., Brunak, S., von Heijne, G., and Nielsen, H. 2011. SignalP 4.0: Discriminating signal peptides from transmembrane regions. Nat Methods 8:785-786.

Rafiqi, M., Ellis, J. G., Ludowici, V. A., Hardham, A. R., and Dodds, P. N. 2012. Challenges and progress towards understanding the role of effectors in plant-fungal interactions. Curr. Opin. Plant Biol. 15:477-482.

Randall, E., Young, V., Sierotzki, H., Scalliet, G., Birch, P. R. J., Cooke, D. E. L., Csukai, M., and Whisson, S. C. 2014. Sequence diversity in the large subunit of RNA polymerase I contributes to mefenoxam insensitivity in Phytophthora infestans. Mol. Plant Pathol. 15:664-676.

Rawlings, N. D., Barrett, A. J., and Bateman, A. 2010. MEROPS: The peptidase database. Nucleic Acids Res. 38:D227-D233.

Revanna, K. V., Munro, D., Gao, A., Chiu, C. C., Pathak, A., and Dong, Q. 2012. A web-based multi-genome synteny viewer for customized data. BMC Bioinformatics 13:190.

Ristaino, J. B., Groves, C. T., and Parra, G. R. 2001. PCR amplification of the Irish potato famine pathogen from historic specimens. Nature 411: 695-697.

Ristaino, J. B., Johnson, A., Blanco-meneses, M., Liu, B., Pathology, P., and Carolina, N. 2007. Identification of the tobacco blue mold pathogen, Peronospora tabacina, by polymerase chain reaction. Plant Dis. 91:685-691.

Rozynek, R., and Spring, O. 2001. Leaf disc inoculation, a fast and precise test for the screening of metalaxyl tolerance in sunflower downy mildew. Phytopathology 149:309-312.

Sastry, J. G., Sivaramakrishnan, S., Thakur, R. P., Gupta, V. S., and Ranjekar, P. K. 1997. Characterization of the genome of Sclerospora graminicola, the causal fungus of downy mildew of pearl millet. World J. Microb. Biot. 13:693-697. 
Saunders, D. G. O., Win, J., Kamoun, S., and Raffaele, S. 2014. Twodimensional data binning for the analysis of genome architecture in filamentous plant pathogens and other eukaryotes. Pages 29-51 in: Methods in Molecular Biology, Vol 1127. P. Birch, J. T.Jones, and J. I. B. Bos, eds. Humana Press, New York.

Schmieder, R., and Edwards, R. 2011. Fast identification and removal of sequence contamination from genomic and metagenomic datasets. PLoS One 6:e17288.

Seidl, M. F., Faino, L., Shi-kunne, X., van den Berg, G. C. M., Bolton, M. D., and Thomma, B. P. H. J. 2015. The genome of the saprophytic fungus Verticillium tricorpus reveals a complex effector repertoire resembling that of its pathogenic relatives. Mol. Plant-Microbe Interact. 28:362-373.

Sievers, F., Wilm, A., Dineen, D., Gibson, T. J., Karplus, K., Li, W., Lopez, R., McWilliam, H., Remmert, M., Söding, J., Thompson, J. D., and Higgins, D. G. 2011. Fast, scalable generation of high-quality protein multiple sequence alignments using Clustal Omega. Mol. Syst. Biol. 7:539. Spencer, D. 1981. The Downy Mildews. Academic Press, London.

Smit, A. F. A., Hubley, R. and Green, P. 2013. RepeatMasker Open-4.0. http://www.repeatmasker.org. Institute for Systems Biology, Seattle, WA, U.S.A.

Spring, O. 2012. Oomycota. Pages 98-105 in: Syllabus of Plant Families. W. Frey, ed. Gebrüder Borntraeger verlagsbuchhandlung, Stuttgart, Germany.

Spring, O., Hammer, T. R., Zipper, R., and Billenkamp, N. 2013. Population dynamics in tobacco blue mold incidences as a consequence of pathogen control and virulence performance of Peronospora tabacina phenotypes. Crop Prot. 45:76-82.

Spurr, H. W., and Todd, F. A. 1919. Oospores in blue mold diseased North Carolina burley and flue-cured tobacco. Tobacco Sci. 184:27-29.

Stanke, M., Steinkamp, R., Waack, S., and Morgenstern, B. 2004. AUGUSTUS: A web server for gene finding in eukaryotes. Nucleic Acids Res. 32:W309-W312.

Stassen, J. H. M., Seidl, M. F., Vergeer, P. W. J., Nijman, I. J., Snel, B., Cuppen, E., and van den Ackerveken, G. 2012. Effector identification in the lettuce downy mildew Bremia lactucae by massively parallel transcriptome sequencing. Mol. Plant Pathol. 13:719-731.

Steinbiss, S., Willhoeft, U., Gremme, G., and Kurtz, S. 2009. Fine-grained annotation and classification of de novo predicted LTR retrotransposons. Nucleic Acids Res. 37:7002-7013.

Strasburg, J. L., and Rieseberg, L. H. 2008. Molecular demographic history of the annual sunflowers Helianthus annuus and $H$. petiolaris-Large effective population sizes and rates of long-term gene flow. Evolution 62:1936-1950.

Sukno, S. A., Taylor, A. M., and Farman, M. L. 2002. Genetic uniformity among isolates of Peronospora tabacina, the tobacco blue mold pathogen. Phytopathology 92:1236-1244.

Suyama, M., Torrents, D., and Bork, P. 2006. PAL2NAL: Robust conversion of protein sequence alignments into the corresponding codon alignments. Nucleic Acids Res. 34:W609-W612.

Thines, M. 2014. Phylogeny and evolution of plant pathogenic oomycetes-A global overview. Eur. J. Plant Pathol. 138:431-447.

Thines, M., Komjáti, H., and Spring, O. 2005. Exceptional length of ITS in Plasmopara halstedii is due to multiple repetitions in the ITS-2 region. Eur. J. Plant Pathol. 112:395-398.

Tian, M., Win, J., Savory, E., Burkhardt, A., Held, M., Brandizzi, F., and Day, B. 2011. 454 Genome sequencing of Pseudoperonospora cubensis reveals effector proteins with a QXLR translocation motif. Mol. PlantMicrobe Interact. 24:543-553.

Torto, T. A., Li, S., Styer, A., Huitema, E., Testa, A., Gow, N. A., van West, P., and Kamoun, S. 2003. EST mining and functional expression assays identify extracellular effector proteins from the plant pathogen Phytophthora. Genome Res. 13:1675-1685.

Trigiano, R. N., Wadl, P. A., Dean, D., Hadziabdic, D., Scheffler, B. E., Runge, F., Telle, S., Thines, M., Ristaino, J., and Spring, O. 2012. Ten polymorphic microsatellite loci identified from a small insert genomic library for Peronospora tabacina. Mycologia 104:633-640.

Tyler, B. M., Tripathy, S., Zhang, X., Dehal, P., Jiang, R. H. Y., Aerts, A., Arredondo, F. D., Baxter, L., Bensasson, D., Beynon, J. L., Chapman, J., Damasceno, C. M. B., Dorrance, A. E., Dou, D., Dickerman, A. W., Dubchak, I. L., Garbelotto, M., Gijzen, M., Gordon, S. G., Govers, F., Grunwald, N. J., Huang, W., Ivors, K. L., Jones, R. W., Kamoun, S., Krampis, K., Lamour, K. H., Lee, M.-K., McDonald, W. H., Medina, M., Meijer, H. J. G., Nordberg, E. K., Maclean, D. J., Ospina-Giraldo, M. D., Morris, P. F., Phuntumart, V., Putnam, N. H., Rash, S., Rose, J. K. C., Sakihama, Y., Salamov, A. A., Savidor, A., Scheuring, C. F., Smith, B. M., Sobral, B. W., Terry, A., Torto-Alalibo, T. A., Win, J., Xu, Z.,
Zhang, H., Grigoriev, I. V., Rokhsar, D. S., and Boore, J. L. 2006. Phytophthora genome sequences uncover evolutionary origins and mechanisms of pathogenesis. Science 313:1261-1266.

Vitte, C., Panaud, O., and Quesneville, H. 2007. LTR retrotransposons in rice (Oryza sativa, L.): Recent burst amplifications followed by rapid DNA loss. BMC Genomics 8:218.

Voglmayr, H. 2003. Phylogenetic relationships of Peronospora and related genera based on nuclear ribosomal ITS sequences. Mycol. Res. 107: 1132-1142.

Voglmayr, H., and Greilhuber, J. 1998. Genome size determination in peronosporales (Oomycota) by Feulgen image analysis. Fungal Genet. Biol. 25:181-195.

Whisson, S. C., Boevink, P. C., Moleleki, L., Avrova, A. O., Morales, J. G., Gilroy, E. M., Armstrong, M. R., Grouffaud, S., van West, P., Chapman, S., Hein, I., Toth, I. K., Pritchard, L., and Birch, P. R. J. 2007. A translocation signal for delivery of oomycete effector proteins into host plant cells. Nature 450:115-118.

Wicker, T., Matthews, D. E., and Keller, B. 2002. TREP: A database for Triticeae repetitive elements. Trends Plant Sci. 7:561-562.

Wicker, T., Sabot, F., Hua-Van, A., Bennetzen, J. L., Capy, P., Chalhoub, B., Flavell, A., Leroy, P., Morgante, M., Panaud, O., Paux, E., SanMiguel, P., and Schulman, A. H. 2007. A unified classification system for eukaryotic transposable elements. Nat. Rev. Genet. 8:973-982.

Wiglesworth, M. D., Reuveni, M., Nesmith, W., C., Siegel, M. R., Kuc, J., Juarez, J., and Tabamex, 1988. Resistance of Peronospora tabacina to metalaxyl in Texas and Mexico. Plant Dis. 72:964-967.

Win, J., and Kamoun, S. 2008. Adaptive evolution has targeted the C-terminal domain of the RXLR effectors of plant pathogenic oomycetes. Plant Signal Behav. 3:251-253.

Win, J., Krasileva, K. V., Kamoun, S., Shirasu, K., Staskawicz, B. J., and Banfield, M. J. 2012. Sequence divergent RXLR effectors share a structural fold conserved across plant pathogenic oomycete species. PLoS Pathog. 8:e1002400.

Win, J., Morgan, W., Bos, J., Krasileva, K. V., Cano, L. M., ChaparroGarcia, A., Ammar, R., Staskawicz, B. J., and Kamoun, S. 2007. Adaptive evolution has targeted the C-terminal domain of the RXLR effectors of plant pathogenic oomycetes. Plant Cell 19:2349-2369.

Wong, J. 2011. Molecular and genetic analysis of plant-pathogen interactions in lettuce and Bremia lactucae. Page 263. Ph.D. thesis, University of California Davis.

Yandell, M., and Ence, D. 2012. A beginner's guide to eukaryotic genome annotation. Nat. Rev. Genet. 13:329-342.

Yang, Z. 2007. PAML 4: Phylogenetic analysis by maximum likelihood. Mol. Biol. Evol. 24:1586-1591.

Zipper, R., Hammer, T. R., and Spring, O. 2009. PCR-based monitoring of recent isolates of tobacco blue mold from Europe reveals the presence of two genetically distinct phenotypes differing in fungicide sensitivity. Eur. J. Plant Pathol. 123:367-375.

\section{AUTHOR-RECOMMENDED INTERNET RESOURCES}

allPrep-8.py python script: http://comailab.genomecenter.ucdavis.edu/index.php/Barcoded_data_ preparation_tools

Batch CD-search tool:

http://www.ncbi.nlm.nih.gov/Structure/bwrpsb/bwrpsb.cgi

bedtools suite v2.17.0: http://bedtools.readthedocs.org/en/latest

BWA-MEM: http://bio-bwa.sourceforge.net

CLC Genomics Workbench v6.5: http://www.clcbio.com

Clustal Omega: http://www.ebi.ac.uk/Tools/msa/clustalo

FastQC: http://www.bioinformatics.babraham.ac.uk/projects/fastqc

hmmsearch program: http://hmmer.janelia.org

Interactive Tree of Life: http://itol.embl.de/

NCBI bacterial genome sequences database:

ftp://ftp.ncbi.nlm.nih.gov/genomes/Bacteria/all.faa.tar.gz

Oomycete Transcriptomics database:

http://www.eumicrobedb.org/transcripts

RepeatModeler v1.0.8: http://www.repeatmasker.org

Scythe: https://github.com/vsbuffalo/scythe

University of California Davis DNA Technologies Core website:

http://genomecenter.ucdavis.edu

University of California Davis genome browsers:

http://gviewer.gc.ucdavis.edu/cgi-bin/gbrowse/Ptabacina_968-J2_V1

http://gviewer.gc.ucdavis.edu/cgi-bin/gbrowse/Ptabacina_968-S26_V1

Vmatch: http://www.vmatch.de 